\title{
Nonisothermal nematic liquid crystal flows with the Ball-Majumdar free energy
}

\author{
Eduard Feireisl \\ Institute of Mathematics of the Czech Academy of Sciences, \\ Žitná 25, 11567 Praha 1, Czech Republic \\ E-mail: feireisl@math.cas.cz * \\ Giulio Schimperna \\ Dipartimento di Matematica, Università di Pavia, \\ Via Ferrata 1, 27100 Pavia, Italy \\ E-mail: giusch04@unipv.it ${ }^{\dagger}$ \\ Elisabetta Rocca \\ Weierstrass Institute for Applied Analysis and Stochastics, \\ Mohrenstr. 39, D-10117 Berlin, Germany \\ E-mail: rocca@wias-berlin.de \\ and \\ Dipartimento di Matematica, Università di Milano, \\ Via Saldini 50, 20133 Milano, Italy \\ E-mail elisabetta.rocca@unimi.it $\ddagger$ \\ Arghir Zarnescu \\ Pevensey III, University of Sussex, \\ Falmer, BN1 9QH, UK \\ E-mail: A.Zarnescu@sussex.ac.uk ${ }^{\S}$
}

\begin{abstract}
In this paper we prove the existence of global in time weak solutions for an evolutionary PDE system modelling nonisothermal Landau-de Gennes nematic liquid crystal (LC) flows in three dimensions of space. In our model, the incompressible Navier-Stokes system for the macroscopic velocity $\mathbf{u}$ is coupled to a nonlinear convective parabolic equation describing the evolution of the Q-tensor $\mathbb{Q}$, namely a tensor-valued variable representing the normalized second order moments of the probability distribution function of the LC molecules. The effects of the (absolute) temperature $\vartheta$ are prescribed in the form of an energy balance identity complemented with a global entropy production inequality. Compared to previous contributions, we can consider here the physically realistic singular configuration potential $f$ introduced by Ball and Majumdar. This potential gives rise to severe mathematical difficulties since it introduces, in the Q-tensor equation, a term which is at the same time singular in $\mathbb{Q}$ and degenerate in $\vartheta$. To treat it a careful analysis of the properties of $f$, particularly of its blow-up rate, is carried out.
\end{abstract}

Key words: nematic liquid crystal, Ball-Majumdar free energy, nonisothermal model, existence theorem.

\footnotetext{
${ }^{*}$ The research of E.F. leading to these results has received funding from the European Research Council under the European Union's Seventh Framework Programme (FP7/2007-2013)/ ERC Grant Agreement 320078. The work of E.F. was partially supported by the FP7-IDEAS-ERC-StG \#256872 (EntroPhase).

${ }^{\dagger}$ The work of G.S. was supported by the FP7-IDEAS-ERC-StG \#256872 (EntroPhase).

${ }^{\ddagger}$ The work of E.R. was supported by the FP7-IDEAS-ERC-StG \#256872 (EntroPhase).

$\S$ The work of A.Z. was partially supported by the FP7-IDEAS-ERC-StG \#256872 (EntroPhase).
} 
AMS (MOS) subject classification: 76A15, 74G25, 35D30, 35Q30.

\section{Introduction}

The main aim of this paper is to continue the analysis of non-isothermal Landau-de Gennes nematic liquid crystal flows with singular potential started in our recent contribution [19]. Our purpose is to consider the evolution of the flow assuming that the mixing term in the bulk potential is singular, and, in particular, takes the form introduced by Ball and Majumdar in [5]:

$$
\psi_{B}(\vartheta, \mathbb{Q})=\vartheta f(\mathbb{Q})+G(\mathbb{Q}),
$$

where $\vartheta$ denotes the absolute temperature and the $Q$-tensor $\mathbb{Q}$ is a symmetric traceless tensor representing in a suitable way the normalized second order moments of the probability distribution function of molecules. Here $G$ is a smooth function of $\mathbb{Q}$ while $f: R_{\mathrm{sym}, 0}^{3 \times 3} \rightarrow[-K,+\infty], K \geq 0$, is a convex function smooth on its domain which consists of $\mathbb{Q}$-tensors whose eigenvalues are inside the physical interval $(-1 / 3,2 / 3)$.

The use of Q-tensors permits to capture fine properties of the crystal configuration ( like biaxiality), which are not possible to describe in the framework of vector-based models. In order to introduce Q-tensors mathematically, we start from a probability measure $\mu_{x}$ on the unit sphere $\mathbb{S}^{2}$, representing the orientation of the molecules at a point $x$ in space. Then, we can associate to $\mu_{x}$ a symmetric and traceless $3 \times 3$ matrix defined as

$$
\mathbb{Q}(x)=\int_{\mathbb{S}^{2}}\left(\mathbf{p} \otimes \mathbf{p}-\frac{1}{3} \mathbb{I}\right) \mathrm{d} \mu_{x}(\mathbf{p}) .
$$

Namely, $\mathbb{Q}$ is intended to measure how much the probability measure $\mu_{x}$ deviates from the isotropic measure $\bar{\mu}$ where $d \bar{\mu}=\frac{1}{4 \pi} d A$, see [15]. In the Onsager model (cf. [15], 31]), $\mu_{x}$ is assumed to be absolutely continuous with density $\rho=\rho(\mathbf{p})$. In this case, we have

$$
\mathbb{Q}(x)=\int_{\mathbb{S}^{2}}\left(\mathbf{p} \otimes \mathbf{p}-\frac{1}{3} \mathbb{I}\right) \rho(\mathbf{p}) \mathrm{d} \mathbf{p} .
$$

The fact that $\mu_{x}$ is a probability measure imposes a constraint on the eigenvalues of $\mathbb{Q}$, namely that they are bounded between the values $-1 / 3$ and $2 / 3$, see $[5$. Thus not any traceless $3 \times 3$ matrix is a physical $\mathbb{Q}$-tensor but only those whose eigenvalues are in $(-1 / 3,2 / 3)$.

The Ball-Majumdar potential is then defined as follows:

$$
\begin{gathered}
f(\mathbb{Q})=\left\{\begin{array}{c}
\inf _{\rho \in \mathcal{A}_{\mathbb{Q}}} \int_{S^{2}} \rho(\mathbf{p}) \log (\rho(\mathbf{p})) \mathrm{d} \mathbf{p} \text { if } \lambda_{i}[\mathbb{Q}] \in(-1 / 3,2 / 3), i=1,2,3, \\
+\infty \text { otherwise },
\end{array}\right. \\
\mathcal{A}_{\mathbb{Q}}=\left\{\rho: S^{2} \rightarrow[0, \infty) \mid \rho \in L^{1}\left(S^{2}\right), \int_{S^{2}} \rho(\mathbf{p}) \mathrm{d} \mathbf{p}=1 ; \mathbb{Q}=\int_{S^{2}}\left(\mathbf{p} \otimes \mathbf{p}-\frac{1}{3} \mathbb{I}\right) \rho(\mathbf{p}) \mathrm{d} \mathbf{p}\right\} .
\end{gathered}
$$

Then, it turns out (see, e.g., [5]) that the effective domain $\mathcal{D}[f]$ (i.e., the set where $f$ assumes finite values) coincides precisely with the set of symmetric traceless tensors whose eigenvalues belong to the physical interval. Moreover, as we prove in detail in Section 4 below, $f$ explodes logarithmically fast as $\mathbb{Q}$ approaches the boundary of $\mathcal{D}[f]$ (or, equivalently, one of its eigenvalues tends to $-1 / 3$ ). The main mathematical features of $f$, which are required in the existence proof for the purpose of obtaining the necessary a-priori estimates, are thoroughly discussed in Section 4 . Here, it is just worth noting that, for large values of the temperature, the convex part $f$ of the energy $\psi_{B}$ is prevailing, whereas, when $\vartheta$ is close to $0, \psi_{B}$ may exhibit a multiple well structure.

Dealing with the bulk potential (1.1) generates severe mathematical difficulties, since the term $\vartheta f(\mathbb{Q})$ is both singular in $\mathbb{Q}$ (since the eigenvalues of $\mathbb{Q}$ may approach, at least in some small region, the limiting values $-1 / 3,2 / 3$, and degenerate in $\vartheta$ (since we can only prove that $\vartheta>0$ almost everywhere, not excluding, however, that the (essential) infimum of $\vartheta$ might reach 0 ). Because of several technical 
problems associated with the above mentioned difficulties, our previous contribution [19] focused on the case when the bulk potential in the free energy functional is given by (cf. also [43] and [33])

$$
\psi_{B}(\vartheta, \mathbb{Q})=f(\mathbb{Q})-U(\vartheta) G(\mathbb{Q}),
$$

where $U$ is assumed to be a convex and decreasing function of $\vartheta$ with restricted growth at $\infty$. This form of the bulk potential allowed us to get rid of the degenerate character in $\vartheta$, and, in fact, to prove strict positivity of $\vartheta$ in that case. Although the formula (1.1) is mathematically equivalent to (1.4) in the framework of stationary problems, they give rise to different solutions if the time evolution of the system is studied. In accordance with the underlying physical arguments (see [31]), it is (1.1) rather than (1.4) that should be considered. Indeed this choice, combined with the standard principles of Thermodynamics, yields the entropy of the system in the form

$$
s=-\frac{\partial \mathcal{F}}{\partial \vartheta}=c(\vartheta)-f(\mathbb{Q}),
$$

where $\mathcal{F}$ denotes the free energy functional, and $c$ represents its purely caloric part (cf. also (1.4)). On the other hand, in the case (1.4), one has

$$
s=c(\vartheta)+U^{\prime}(\vartheta) G(\mathbb{Q}),
$$

and the singular potential $f$ appears only in the internal energy of the system.

In our model, the evolution of the Q-tensor is derived as a balance of the following free energy functional:

$$
\mathcal{F}\left(\mathbb{Q}, \nabla_{x} \mathbb{Q}, \vartheta\right)=\frac{1}{2}\left|\nabla_{x} \mathbb{Q}\right|^{2}+\psi_{B}(\vartheta, \mathbb{Q})-\vartheta \log \vartheta-a \vartheta^{m},
$$

where $\lambda, a \geq 0$ and the exponent $m \geq 1$ will be fixed below. The term depending on $\nabla_{x} \mathbb{Q}$ describes the interfacial energy, whereas $a \vartheta^{m}, a>0$, prescribes a power-like heat conductivity.

The macroscopic velocity of the crystal flow is described by a Navier-Stokes type system (cf. (2.3) below) for the velocity field $\mathbf{u}$, in which the stress tensor contains a non-standard part depending on the tensor $\mathbb{Q}$. The evolution of $\mathbb{Q}(\mathrm{cf}$. (2.5) $)$ is ruled by the variational derivative of the free-energy functional, displaying a singular character due to the presence of the singular BallMajumdar energy (1.1). In the physical formulation of the model, the evolution of temperature is described by the heat equation (2.8). However, due to the presence of quadratic terms in the right hand side (especially those depending on $\mathbf{u}$ ), dealing with (2.8) appears out of reach from a mathematical perspective. For this reason, following an idea originally developed by Bulíček, Feireisl and Málek in [12] for the non-isothermal Navier-Stokes system, we replace it by an equality describing conservation of total energy conservation, combined with a weak form of the entropy inequality (cf. (2.17) and (2.23) below). As a drawback of this choice, we have to note the explicit appearence of the pressure $p$ in the energy balance equation. Actually, $p$ is no longer just a Lagrange multiplier, but it enters the system as an additional unknown and needs to be controlled carefully. In particular, for this purpose a suitable choice of the boundary condition is crucial and, indeed, in order to reduce complications to the minimum, we consider periodic b.c.'s (cf. (2.1) below).

Due to the quoted mathematical difficulties, the results we obtain in the present contribution are weaker than those we got in [19]. Actually, here we consider a weaker notion of solution, where the entropy inequality holds only in its integrated (both in space ad in time) form; in other words, we can only prove a global balance of entropy. In addition, in order to deal with the Ball-Majumdar energy (1.1), we need a power-like growth assumptions imposed on the heat conductivity, the specific heat and the collective rotational viscosity coefficient in the system. Mathematically speaking, these assumptions are enforced by the need of sufficiently strong $a$-priori bounds for the temperature $\vartheta$. The derivation of suitable a priori estimates as well as a rigorous justification of compactness of the family of solutions represents the main novelty of the present paper with respect to [19], while the specific construction of suitable approximate solutions, carried over in detail in [19], is a routine matter. This is the reason why we restrict our discussion to the passage from a priori bounds to compactness (sequential stability), leaving the necessary modifications of the construction of suitable approximate solutions in [19] to the interested reader.

As an additional result, in the last part of the paper we see that if we additionally assume that the heat flux is singular as the absolute temperature $\vartheta$ approaches 0 (see Section 5 below), then 
we can recover the entropy inequality in the usual distributional sense. Namely, we can estimate the entropy growth on any region of the space domain, and not only globally. Moreover, we can deal with an even more general class of singular potentials. It is worth noting that taking this type of singular flux law is not only a mathematically ad-hoc assumption, but is a common choice in several types of phase-transition and phase-separation models both in liquids and in crystalline solids (cf., e.g., [14, [39], and 37. where similar growth conditions are assumed). Regarding the choice of power-growing specific heat (cf. the last summand in (1.5)), let us mention the papers [1, 32 for examples in phase transitions and [9, 38, 41] for examples in thermoelastic systems, where this behaviour is observed. Note that, however, in our contribution we can allow the viscosity coefficient to depend on the absolute temperature in a quite general way: it has to be only bounded from below and above without any specific growth condition.

We conclude this introduction by a brief review of other LC models based either on the tensorial variable $\mathbb{Q}$ or other parameters. To begin, a vectorial quantity $\mathbf{d}$ could be used instead of $\mathbb{Q}$ in order to describe the preferred orientation of the molecules at any point (cf., e.g., [13, 17, 29] and references therein). Moreover, we can quote the standard hydrodynamic theories, where the case of regular bulk potential

$$
\psi_{B}(\mathbb{Q})=\frac{a}{2} \operatorname{tr}\left(\mathbb{Q}^{2}\right)-\frac{b}{2} \operatorname{tr}\left(\mathbb{Q}^{3}\right)+\frac{c}{4} \operatorname{tr}^{2}\left(\mathbb{Q}^{2}\right)
$$

is considered (cf. [35] and [36] for the analysis of the corresponding isothermal evolution), it is not clear whether this physical contraint on the eigenvectors is preserved or not, while the choice of the Ball-Majumdar free energy (1.1) naturally enforces this constraint. Let us notice that in the literature there are few papers dealing with liquid crystal models with singular potential. In the isothermal case, we can quote the very recent contribution [46, where existence and regularity of solution in the 3D and 2D cases are obtained for a tensorial model with Ball-Majumdar potential. Regarding the nonisothermal tensorial case, up to our knowledge, the only contribution, in the case of singular potential (1.4), was given in the paper [19, which we already mentioned in this Introduction. Two attempts of considering the non-isothermal case were made in [22] and [18] and refer to vectorial models. In particular, in 18 the stretching and rotation effects of the director field induced by the straining of the fluid were considered and the existence of global in time weak solutions was obtained for the corresponding initial boundary value problem.

Plan of the paper. The remainder of the paper is organized as follows: in Section 2 we first introduce the equations of our mathematical model in the strong (physical) form (Subsection 2.1); then, we specify our basic assumptions on coefficients and data (Subsection 2.2). This permits us to introduce (Subsection 2.3) a weak formulation of the initial-boundary value problem for our model and to present a rigorous existence theorem (Theorem 2.2) of weak solutions. The proof is carried out in Section 3 and is subdivided into several steps: we first prove (Subsection 3.1) that any weak solution complies, at least formally, with a number of a-priori estimates; subsequently, these bounds are shown to be sufficient to provide weak sequential stability (Subsection 3.2). Namely, sequences of solutions starting from (suitably) bounded families of initial data admit weak limits which still solve the problem. A key ingredient in the proof is represented by some fine properties of the Ball-Majumdar potential which are obtained separately in Section 4. Finally, in Section 5 we show that, assuming a singular heat flux law, even more general classes of potentials can be treated and, moreover, the entropy inequality holds in a stronger sense.

\section{The mathematical problem}

\subsection{The equations of the model}

In this part, we write the equations of our mathematical model describing the evolution of the unknown fields $\mathbf{u}$ (macroscopic velocity), $\mathbb{Q}$ (Q-tensor) and $\vartheta$ (absolute temperature). The model can be physically derived by following closely the argument reported in [19, Section 1]. Indeed, the main difference is represented here by the choice of the Ball-Majumdar potential (1.1). However, this does not affect the way the model is obtained, but only the outcoming form of the equations. These are 
stated here in their strong form. In Section 2.3, we will see that, in order to have a mathematically tractable system, passing to a weak formulation is actually necessary.

In order to avoid complications related to interactions with boundaries, we take periodic boundary conditions for all unknowns. Namely, we assume the system be settled in the three dimensional torus

$$
\Omega:=\left(\left.[-\pi, \pi]\right|_{\{-\pi, \pi\}}\right)^{3} .
$$

As already noted in the Introduction, the above choice is crucial for our existence theorem.

The first two relations in our model describe the evolution of the macroscopic velocity $\mathbf{u}$ :

\section{INCOMPRESSIBILITY:}

$$
\operatorname{div}_{x} \mathbf{u}=0 .
$$

\section{MOMENTUM EQUATION:}

$$
\partial_{t} \mathbf{u}+\operatorname{div}_{x}(\mathbf{u} \otimes \mathbf{u})=\operatorname{div}_{x} \sigma .
$$

Here, $\sigma$ denotes the stress tensor, given by

$$
\begin{gathered}
\sigma=\mu(\vartheta)\left(\nabla_{x} \mathbf{u}+\nabla_{x}^{t} \mathbf{u}\right)-p \mathbb{I} \\
+2 \xi(\mathbb{H}: \mathbb{Q})\left(\mathbb{Q}+\frac{1}{3} \mathbb{I}\right)-\xi\left[\mathbb{H}\left(\mathbb{Q}+\frac{1}{3} \mathbb{I}\right)+\left(\mathbb{Q}+\frac{1}{3} \mathbb{I}\right) \mathbb{H}\right]+(\mathbb{Q} \mathbb{H}-\mathbb{H} \mathbb{Q})-\nabla_{x} \mathbb{Q} \odot \nabla_{x} \mathbb{Q},
\end{gathered}
$$

$\xi$ being a fixed scalar parameter, measuring the ratio between the rotation and the aligning effect that a shear flow exerts over the directors, see Beris and Edwards [6].

The behavior of the Q-tensor is ruled by the following relation:

\section{ORDER PARAMETER EVOLUTION:}

$$
\partial_{t} \mathbb{Q}+\mathbf{u} \cdot \nabla_{x} \mathbb{Q}-\mathbb{S}\left(\nabla_{x} \mathbf{u}, \mathbb{Q}\right)=\Gamma(\vartheta) \mathbb{H} .
$$

The nonnegative function $\Gamma(\vartheta)$ represents a collective rotational viscosity coefficient, whereas

$$
\mathbb{S}\left(\nabla_{x} \mathbf{u}, \mathbb{Q}\right)=(\xi \varepsilon(\mathbf{u})+\omega(\mathbf{u}))\left(\mathbb{Q}+\frac{1}{3} \mathbb{I}\right)+\left(\mathbb{Q}+\frac{1}{3} \mathbb{I}\right)(\xi \varepsilon(\mathbf{u})-\omega(\mathbf{u}))-2 \xi\left(\mathbb{Q}+\frac{1}{3} \mathbb{I}\right)\left(\mathbb{Q}: \nabla_{x} \mathbf{u}\right)
$$

denotes stretching and rotation effects on the Q-tensor driven by the macroscopic flow, with $\omega(\mathbf{u})$ standing for the antisymmetric part of the viscous stress, i.e.,

$$
\omega(\mathbf{u})=\frac{1}{2}\left(\nabla_{x} \mathbf{u}-\nabla_{x}^{t} \mathbf{u}\right)
$$

and $\varepsilon(\mathbf{u})$ for its symmetric part, i.e.,

$$
\varepsilon(\mathbf{u})=\frac{1}{2}\left(\nabla_{x} \mathbf{u}+\nabla_{x}^{t} \mathbf{u}\right) .
$$

Finally, $-\mathbb{H}$ indicates the first variation of the free energy $\mathcal{F}$ with respect to $\mathbb{Q}$, namely

$$
\mathbb{H}=\Delta \mathbb{Q}-\vartheta \mathcal{L}\left[\frac{\partial f(\mathbb{Q})}{\partial \mathbb{Q}}\right]+\lambda \mathbb{Q}, \quad \lambda \geq 0,
$$


where we have chosen $G(Q)=-\lambda Q$ in (1.1). The operator $\mathcal{L}[\cdot]$ denotes projection on the space of traceless tensor, that is $\mathcal{L}[\mathbb{M}]=\mathbb{M}-\frac{1}{3} \operatorname{tr}(\mathbb{M})$ for $\mathbb{M} \in R^{3 \times 3}$. In other words, the expression $\mathcal{L}\left[\frac{\partial f(\mathbb{Q})}{\partial \mathbb{Q}}\right]$ represents the subdifferential of $f$, with respect to the structure of the space of traceless tensors, evaluated at the point $\mathbb{Q}$. Finally, the evolution of temperature is represented in the strong formulation by the

\section{HEAT EQuATion:}

$$
\begin{aligned}
\partial_{t} \vartheta & +a(m-1) \partial_{t} \vartheta^{m}+\mathbf{u} \cdot \nabla_{x} \vartheta+a(m-1) \mathbf{u} \cdot \nabla_{x} \vartheta^{m}-\operatorname{div}_{x}\left(\kappa(\vartheta) \nabla_{x} \vartheta\right) \\
& =\vartheta\left(\partial_{t} f(\mathbb{Q})+\mathbf{u} \cdot \nabla_{x} f(\mathbb{Q})\right)+\frac{\mu(\vartheta)}{2}\left|\nabla_{x} \mathbf{u}+\nabla_{x}^{t} \mathbf{u}\right|^{2}+\Gamma(\vartheta)|\mathbb{H}|^{2} .
\end{aligned}
$$

The model can be derived from the free energy functional (1.5) by stating the balances of total energy and of entropy. The procedure is completely analogous to that performed in 19, Section 1.3], to which we refer the reader for details. We just note here that (2.8) corresponds to assuming the internal energy flux being given by (cf. [19, formula (1.30)])

$$
\mathbf{q}=-\kappa(\vartheta) \nabla_{x} \vartheta-\nabla_{x} \mathbb{Q}: \mathbb{S}\left(\nabla_{x} \mathbf{u}, \mathbb{Q}\right) .
$$

\subsection{Assumptions on coefficients and data}

We state here our basic assumptions on coefficients and nonlinear terms in system (2.2)-(2.9), as well as on the initial data. First of all, we ask the viscosity $\mu$ to be bounded with respect to $\vartheta$, while we prescribe a power-like law for the heat flux and a linear growth of the diffusion coefficient $\Gamma$ at infinity:

$$
\begin{aligned}
& \mu \in C^{1}(\mathbb{R} ; \mathbb{R}), \quad 0<\underline{\mu} \leq \mu(r) \leq \bar{\mu} \text { for all } r \in \mathbb{R}, \\
& \kappa(r)=A_{0}+A_{k} r^{k}, \quad A_{0}, A_{k}>0 \\
& \Gamma(r)=\Gamma_{0}+\Gamma_{1} r, \quad \Gamma_{0}, \Gamma_{1}>0 .
\end{aligned}
$$

In addition to these, we need a power-like heat conductivity in equation (2.8). Namely, we take $a>0$ and assume that the exponents $k$ in (2.11) and $m$ in (2.8) are strictly positive and satisfy the relation

$$
A:=\frac{3 k+2 m}{3}>9, \quad \frac{3}{2}<m \leq \frac{6 k}{5} .
$$

Next, we let the initial data $\mathbf{u}_{0}, \mathbb{Q}_{0}$, and $\vartheta_{0}$ satisfy

$$
\begin{aligned}
& \mathbf{u}_{0} \in L^{2}\left(\Omega ; R^{3}\right), \operatorname{div}_{x} \mathbf{u}_{0}=0, \\
& \mathbb{Q}_{0} \in H^{1}\left(\Omega ; R_{\mathrm{sym}, 0}^{3 \times 3}\right), f\left(\mathbb{Q}_{0}\right) \in L^{1}(\Omega), \\
& \vartheta_{0} \in L^{\infty}(\Omega), \operatorname{ess} \inf _{\Omega} \vartheta_{0}=: \underline{\vartheta}>0 .
\end{aligned}
$$

Finally, we assume that $f$ satisfies at least the following basic properties:

(i) $f: R_{\mathrm{sym}, 0}^{3 \times 3} \rightarrow[-K,+\infty]$ is convex and lower semi-continuous, with $K \geq 0$.

(ii) The domain of $f$,

$$
\mathcal{D}[f]=\left\{\mathbb{Q} \in R_{\mathrm{sym}, 0}^{3 \times 3} \mid f(\mathbb{Q})<+\infty\right\}=\left\{\mathbb{Q} \in R_{\mathrm{sym}, 0}^{3 \times 3} \mid \lambda_{i}[\mathbb{Q}] \in(-1 / 3,2 / 3)\right\},
$$

is an open, convex, and bounded subset of $R_{\mathrm{sym}, 0}^{3 \times 3}$.

(iii) $f$ is smooth in $\mathcal{D}[f], \lambda \geq 0$. 
We will see in Section 4 below (cf. also [5, Section 3]) that (i)-(iii) hold in the case when $f$ is given by the Ball-Majumdar potential introduced in (1.3). This is a nontrivial issue since relation (1.3) describes $f$ implicitly in terms of the orientation distribution $(\mathrm{s}) \rho(\mathbf{p})$ generating $\mathbb{Q}$. Hence, the properties of $f$ as a function of $\mathbb{Q}$ need to be properly demonstrated. On the other hand, (i)-(iii) do not characterize the Ball-Majumdar potential completely and are satisfied by a much wider class of convex singular functions. Indeed, in the proof we will need that $f$ satisfies some finer properties, especially related to its blow up rate as $\mathbb{Q}$ approaches the boundary of $\mathcal{D}[f]$. The discussion of these properties, and the proof of the fact that they are satisfied by the Ball-Majumdar singular potential, are postponed to Section 4 .

\subsection{Weak formulation and main results}

A proof of existence of global-in-time strong solutions of the model introduced in Section 2.1 appears to be out of reach for the available mathematical tools, one of the principal difficulties being related to the presence of quadratic terms on the right hand side of the heat equation (2.8). Accordingly, we introduce here a weak formulation of the system along the lines of [19]. The main point stands in the replacement of (2.8) by means of a couple of relations, namely the total energy balance (cf. (2.17) below) and a weak form of the entropy production inequality (cf. (2.23)).

Such relations account for most of the information coming from the heat equation, but are more treatable mathematically. Actually, (2.17) contains no quadratic terms, while inequality (2.23) just states that the global entropy of the system needs to grow (at least) as prescribed by the right hand side. As noted in the Introduction, differently from what was done in [19] and due to technical difficulties related to the energy (1.1), we are not able to get here any "pointwise" control of the entropy (actually, this can be reached under different assumptions, cf. Section 5 below), but we just get a "global" (i.e., integrated) version of the entropy inequality.

Moreover, as a further drawback of this procedure, we note the explicit appearance of the pressure $p$ in (2.17), which explicitly enters the system as an unknown. To get a control of it from the momentum equation, specific choices of the boundary conditions are needed (cf. [20] for further comments on this point). In particular, this can be reached in our case of periodic conditions. These being said, we can state the

\section{TOTAL ENERGY BALANCE:}

$$
\partial_{t}\left(\frac{1}{2}|\mathbf{u}|^{2}+e\right)+\operatorname{div}_{x}\left(\left(\frac{1}{2}|\mathbf{u}|^{2}+e\right) \mathbf{u}\right)+\operatorname{div}_{x} \mathbf{q}=\operatorname{div}_{x}(\sigma \mathbf{u})+\operatorname{div}_{x}\left(\Gamma(\vartheta) \nabla_{x} \mathbb{Q}: \mathbb{H}\right)
$$

where

$$
\operatorname{div}_{x} \mathbf{q}=-\Delta \widehat{\kappa}(\vartheta)-\operatorname{div}_{x}\left(\nabla_{x} \mathbb{Q}: \mathbb{S}\left(\nabla_{x} \mathbf{u}, \mathbb{Q}\right)\right), \quad \widehat{\kappa}(\vartheta)=A_{0} \vartheta+\frac{A_{k}}{k+1} \vartheta^{k+1}
$$

In (2.17), we have noted as $e$ the internal energy

$$
e=\mathcal{F}+\vartheta s=\frac{1}{2}\left|\nabla_{x} \mathbb{Q}\right|^{2}-\frac{\lambda}{2}|\mathbb{Q}|^{2}+\vartheta+a(m-1) \vartheta^{m},
$$

where the entropy $s$ is given by

$$
s=-\frac{\partial \mathcal{F}}{\partial \vartheta}=1+\log \vartheta-f(\mathbb{Q})+m a \vartheta^{m-1} .
$$

Recall also that the stress $\sigma$ is given by (2.4).

If one starts from the strong formulation, then (2.17) is (formally) obtained by multiplying (2.3) by $\mathbf{u},(2.5)$ by $\mathbb{H}$, and summing the results to (2.8). This procedure involves a number of lengthy, but otherwise straighforward, computations, which are provided in detail in [19, Section 1]. In particular, one uses the identity

$$
-\mathbb{H}: \mathbb{S}\left(\nabla_{x} \mathbf{u}, \mathbb{Q}\right)
$$




$$
=(\mathbb{Q} \mathbb{H}-\mathbb{H} \mathbb{Q}): \nabla_{x} \mathbf{u}+2 \xi(\mathbb{H}: \mathbb{Q})\left(\mathbb{Q}: \nabla_{x} \mathbf{u}\right)-\xi\left[\mathbb{H}\left(\mathbb{Q}+\frac{1}{3} \mathbb{I}\right)+\left(\mathbb{Q}+\frac{1}{3} \mathbb{I}\right) \mathbb{H}\right]: \nabla_{x} \mathbf{u},
$$

which holds for any symmetric matrix $\mathbb{H}$.

Finally, multiplying the heat equation (2.8) by $1 / \vartheta$, one readily deduces the "pointwise version" of the entropy production inequality:

$$
\begin{gathered}
\partial_{t} s+\operatorname{div}_{x}(s \mathbf{u})-\operatorname{div}_{x}\left(\frac{\kappa(\vartheta)}{\vartheta} \nabla_{x} \vartheta\right) \\
\geq \frac{1}{\vartheta}\left(\frac{\mu(\vartheta)}{2}\left|\nabla_{x} \mathbf{u}+\nabla_{x}^{t} \mathbf{u}\right|^{2}+\Gamma(\vartheta)|\mathbb{H}|^{2}+\frac{\kappa(\vartheta)}{\vartheta}\left|\nabla_{x} \vartheta\right|^{2}\right) .
\end{gathered}
$$

As noted above, in our existence result we are not able to deal with relation (2.22), even interpreted as a distributional inequality. Indeed, an $L^{p}$-estimate for the term $s \mathbf{u}$ on the left hand side seems out of reach due to the occurrence of the singular function $f(\mathbb{Q})$ as a summand in $s$ (cf. $(2.20)$ ). Hence, we have to replace (2.22), by the following "global" entropy balance (simply obtained integrating (2.22) both in space and in time):

\section{INTEGRATED ENTROPY PRODUCTION INEQUALITY:}

$$
\int_{0}^{t} \int_{\Omega} \frac{1}{\vartheta}\left(\frac{\mu(\vartheta)}{2}\left|\nabla_{x} \mathbf{u}+\nabla_{x}^{t} \mathbf{u}\right|^{2}+\Gamma(\vartheta)|\mathbb{H}|^{2}+\frac{\kappa(\vartheta)}{\vartheta}\left|\nabla_{x} \vartheta\right|^{2}\right)-\int_{\Omega}(s(t)-s(0)) \leq 0, \quad \text { for a.e. } t \in(0, T) \text {. }
$$

We are now ready to detail the notion of weak solution to our problem. Recall that the exponent $A$ was introduced in (2.13).

Definition 2.1. A weak solution to the non-isothermal liquid crystal system with the Ball-Majumdar potential is a quadruplet $(\mathbf{u}, p, \mathbb{Q}, \vartheta)$ enjoying the regularity properties

$$
\begin{aligned}
& \mathbf{u} \in L^{\infty}\left(0, T ; L^{2}\left(\Omega ; \mathbb{R}^{3}\right)\right), \quad \nabla_{x} \mathbf{u} \in L^{\frac{2 A}{A+1}}\left((0, T) \times \Omega ; \mathbb{R}^{3 \times 3}\right), \\
& p \in L^{3 / 2}((0, T) \times \Omega), \\
& \mathbb{Q} \in L^{\infty}\left(0, T ; H^{1}\left(\Omega ; R_{\mathrm{sym}, 0}^{3 \times 3}\right)\right) \cap L^{2}\left(0, T ; H^{2}\left(\Omega ; R_{\mathrm{sym}, 0}^{3 \times 3}\right)\right), \quad \mathbb{Q}_{t} \in L^{\frac{12}{7}}\left((0, T) \times \Omega ; R_{\mathrm{sym}, 0}^{3 \times 3}\right), \\
& \vartheta \in L^{A}((0, T) \times \Omega), \vartheta>0 \text { a.e. in }(0, T) \times \Omega, \\
& \log \vartheta \in L^{\infty}\left(0, T ; L^{1}(\Omega)\right) \cap L^{2}\left(0, T ; H^{1}(\Omega)\right), \\
& \frac{1}{2}|\mathbf{u}|^{2}+e \in C_{w}([0, T] ; X),
\end{aligned}
$$

where $X$ is a Sobolev space of negative order depending on $k$ and $m$, satisfying the incompressibility condition (2.2), the momentum equation (2.3), the Q-tensor evolution system (2.5) and the total energy balance (2.17) at least in the sense of distributions, and complying with the initial conditions in the following sense:

$$
\begin{aligned}
& \left.\mathbf{u}\right|_{t=0}=\mathbf{u}_{0},\left.\quad \mathbb{Q}\right|_{t=0}=\mathbb{Q}_{0}, \\
& \left.\left(\frac{1}{2}|\mathbf{u}|^{2}+e\right)\right|_{t=0}=\left(\frac{1}{2}\left|\mathbf{u}_{0}\right|^{2}+e_{0}\right)
\end{aligned}
$$

where

$$
e_{0}:=\frac{1}{2}\left|\nabla_{x} \mathbb{Q}_{0}\right|^{2}-\frac{\lambda}{2}\left|\mathbb{Q}_{0}\right|^{2}+\vartheta_{0}+a(m-1) \vartheta_{0}^{m}
$$

and $e$ is defined in (2.19). 
Then, our existence theorem, which can be considered as the main result of this paper, reads

Theorem 2.2. Under the assumptions stated in Subsection 2.2, the non-isothermal liquid crystal model with Ball-Majumdar potential admits at least one weak solution, which additionally satisfies the integrated entropy production inequality (2.23).

The main steps of the proof of the theorem are carried out in the next section.

Remark 2.3. In the statement, we claimed that the system equations hold at least in the sense of distributions. To be more precise, we can say that the momentum equation holds in the form

$$
\int_{0}^{T} \int_{\Omega}\left[\mathbf{u} \cdot \partial_{t} \varphi+(\mathbf{u} \otimes \mathbf{u}): \nabla_{x} \varphi\right]=\int_{0}^{T} \int_{\Omega} \sigma: \nabla_{x} \varphi-\int_{\Omega} \mathbf{u}_{0} \cdot \varphi(0, \cdot),
$$

satisfied for any test function $\varphi \in C_{c}^{\infty}\left([0, T) \times \Omega ; R^{3}\right)$. On the other hand, by virtue of the regularity properties (2.24)-(2.28), the order parameter equation (2.5) turns out to hold pointwise (i.e. in some $L^{p}$-space) and the periodic b.c. for $\mathbb{Q}$ hold in the sense of traces. Finally, the total energy balance (2.17) is satisfied as the integral identity

$$
\begin{gathered}
\int_{0}^{T} \int_{\Omega}\left[\left(\frac{1}{2}|\mathbf{u}|^{2}+e\right) \partial_{t} \varphi+\left(\frac{1}{2}|\mathbf{u}|^{2}+e\right) \mathbf{u} \cdot \nabla_{x} \varphi+\widehat{\kappa}(\vartheta) \Delta \varphi-\left(\nabla_{x} \mathbb{Q}: \mathbb{S}\left(\nabla_{x} \mathbf{u}, \mathbb{Q}\right)\right) \cdot \nabla_{x} \varphi\right] \\
=\int_{0}^{T} \int_{\Omega}\left[\sigma \mathbf{u} \cdot \nabla_{x} \varphi+\Gamma(\vartheta)\left(\nabla_{x} \mathbb{Q}: \mathbb{H}\right) \cdot \nabla_{x} \varphi\right]-\int_{\Omega}\left(\frac{1}{2}\left|\mathbf{u}_{0}\right|^{2}+e_{0}\right) \varphi(0, \cdot)
\end{gathered}
$$

for any $\varphi \in C_{c}^{\infty}([0, T) \times \Omega)$, where $e$ was defined in (2.19) and $\widehat{\kappa}$ was introduced in (2.18). Actually, we needed to substitute the expression (2.9) for $\mathbf{q}$ and manage it by a further integration by parts.

Finally, let us note here that in Section 5 we will state another existence result (Thm. 5.1) holding in case of singular heat flux law (5.1) and in that case the entropy inequality holds true in the usual distributional form (cf. (5.5)).

\section{Proof of Theorem 2.2}

The remainder of the paper is devoted to the proof of Theorem 2.2. The argument we are going to perform follows closely the lines of the proof given in our previous work [19, the main difference being represented of course by the presence of the Ball-Majumdar potential. In order to reduce complications, we will avoid describing in detail the (rather long and technical) approximation procedure needed to get an existence theorem for a regularized version of the system. Indeed, at this level, we do not see relevant differences with respect to the argument given in [19, to which we refer the reader for more details.

Instead, the subsequent part of the proof is more significantly different from that in 19 and, for this reason, will be presented in an extended way. As a first step, we will prove in Subsection 3.1 that any weak solution satisfies (at least formally) a number of a-priori estimates, mainly coming as direct consequences of the energy and entropy balances. In particular, we will get a control of certain norms of the solution components only in terms of the initial data and of the fixed parameters of the system. These basic estimates, however, do not provide any bound on the (subdifferential of the) singular term $f$. Its control is actually based on a rather delicate argument detailed in Section 4 and concerning estimates of its Hessian. This part is the key point of our argument and represents the main novelty of the present paper.

In addition to that, we have to stress that, compared to [19, we have here different (powerlike) growth conditions on the viscosity coefficient $\Gamma(\vartheta)$, on the heat conductivity (cf. (2.11)), and on the specific heat (cf. (1.5)). Mathematically speaking, these are needed in order to control some 
$L^{p}$-norm of $\vartheta$. However, in this case, we can allow for a general dependence of the viscosity coefficient from the absolute temperature (cf. (2.10) ).

With the a-priori estimates at our disposal, we will prove in Subsection 3.2 that any sequence $\left(\mathbf{u}_{n}, p_{n}, \mathbb{Q}_{n}, \vartheta_{n}\right)$ which complies with the bounds uniformly with respect to $n$ admits a limit point which is still a weak solution of the system. This part, which we call "weak sequential stability" of weak solutions, can be viewed as a simplified version of the compactness argument needed to remove some regularization of approximation of the system (like that provided in [19]).

\subsection{A priori estimates}

In this section, we perform formal a priori estimates. As noted above, these estimates can be rigorously justified for certain approximate solutions in the way detailed in [19. Most of the bounds follow directly from the total energy conservation (2.17) and the entropy inequality (2.22) (indeed, the integrated version (2.23), holding for weak solutions, suffices for our purposes).

Energy-entropy estimate. Integrating (2.17) in space and using (2.19) with the periodic boundary conditions, we readily obtain the energy estimate

$$
\frac{\mathrm{d}}{\mathrm{d} t} \int_{\Omega}\left(\frac{1}{2}|\mathbf{u}|^{2}+e\right)=\frac{\mathrm{d}}{\mathrm{d} t} \int_{\Omega}\left(\frac{1}{2}|\mathbf{u}|^{2}+\frac{1}{2}\left|\nabla_{x} \mathbb{Q}\right|^{2}-\frac{\lambda}{2}|\mathbb{Q}|^{2}+\vartheta+a(m-1) \vartheta^{m}\right)=0 .
$$

Due to lack of coercivity of the internal energy w.r.t. $\mathbb{Q}$, the above information has to be coupled with the bound coming from the entropy inequality (2.23). Actually, recalling (2.20) and (2.22), we infer

$$
\frac{\mathrm{d}}{\mathrm{d} t} \int_{\Omega}\left(-\log \vartheta-m a \vartheta^{m-1}+f(\mathbb{Q})\right)+\int_{\Omega}\left(\frac{\mu(\vartheta)}{2 \vartheta}\left|\nabla_{x} \mathbf{u}+\nabla_{x}^{t} \mathbf{u}\right|^{2}+\frac{\Gamma(\vartheta)}{\vartheta}|\mathbb{H}|^{2}+\frac{\kappa(\vartheta)}{\vartheta^{2}}\left|\nabla_{x} \vartheta\right|^{2}\right) \leq 0 .
$$

Summing (3.1) to (some positive constant times) (3.2), we immediately obtain the estimates

$$
\begin{aligned}
& \|\mathbf{u}\|_{L^{\infty}\left(0, T ; L^{2}\left(\Omega ; \mathbb{R}^{3}\right)\right)} \leq c, \\
& \|\log \vartheta\|_{L^{\infty}\left(0, T ; L^{1}(\Omega)\right)} \leq c, \\
& \|\vartheta\|_{L^{\infty}\left(0, T ; L^{m}(\Omega)\right)} \leq c, \\
& \|f(\mathbb{Q})\|_{L^{\infty}\left(0, T ; L^{1}(\Omega)\right)} \leq c, \\
& \|\mathbb{Q}\|_{L^{\infty}\left(0, T ; H^{1}(\Omega)\right)} \leq c .
\end{aligned}
$$

In particular, we used here the singular character of $f$, which also gives

$$
\|\mathbb{Q}\|_{L^{\infty}\left((0, T) \times \Omega ; R_{\mathrm{sym}, 0}^{3 \times 3}\right.} \leq c .
$$

Moreover, we have the information coming from the latter integral in (3.2), which we now specify.

First of all, using (2.11), we readily infer

$$
\left\|\nabla_{x} \vartheta^{\kappa / 2}\right\|_{L^{2}\left(0, T ; L^{2}\left(\Omega ; R^{3}\right)\right)}+\left\|\nabla_{x} \log \vartheta\right\|_{L^{2}\left(0, T ; L^{2}\left(\Omega ; R^{3}\right)\right)} \leq c .
$$

Combining (3.4), the second part of (3.9) and using some generalized version of Poincaré's inequality (see, e.g., [42, Lemma 3.2]), we get

$$
\|\log \vartheta\|_{L^{2}\left(0, T ; H^{1}(\Omega)\right)} \leq c .
$$

Next, by (3.5), (3.9) and interpolation, recalling also (2.13), it is easy to infer

$$
\|\vartheta\|_{L^{A}((0, T) \times \Omega)}=\|\vartheta\|_{L^{\frac{3 k+2 m}{3}}((0, T) \times \Omega)} \leq c .
$$

Next, by virtue of (2.10) and (2.12), we obtain

$$
\begin{aligned}
& \left\|\vartheta^{-1 / 2} \nabla_{x} \mathbf{u}\right\|_{L^{2}\left(0, T ; L^{2}\left(\Omega ; \mathbb{R}^{3 \times 3}\right)\right)} \leq c, \\
& \|\mathbb{H}\|_{L^{2}\left(0, T ; L^{2}\left(\Omega ; R_{\mathrm{sym}, 0}^{3 \times 3}\right)\right)}+\left\|\vartheta^{-1 / 2} \mathbb{H}\right\|_{L^{2}\left(0, T ; L^{2}\left(\Omega ; R_{\mathrm{sym}, 0}^{3 \times 3}\right)\right)} \leq c .
\end{aligned}
$$


As a consequence, by (3.11), (3.12) and interpolation, we have

$$
\left\|\nabla_{x} \mathbf{u}\right\|_{L^{\frac{2 A}{A+1}}\left((0, T) \times \Omega ; \mathbb{R}^{3 \times 3}\right)} \leq c,
$$

whence, also,

$$
\|\mathbf{u}\|_{L^{\frac{2 A}{A+1}}\left(0, T ; L^{\frac{6 A}{A+3}}\left(\Omega ; \mathbb{R}^{3}\right)\right)} \leq c\|\mathbf{u}\|_{L^{\frac{2 A}{A+1}}\left(0, T ; W^{1,} \frac{2 A}{A+1}\left(\Omega ; \mathbb{R}^{3}\right)\right)} \leq c .
$$

Interpolating between (3.3) and (3.15) and using that $A>9$ (cf. (2.13) $)$, we arrive at

$$
\left\||\mathbf{u}|^{3}\right\|_{L^{1+}((0, T) \times \Omega)} \leq c .
$$

Here $1+$ denotes a generic exponent strictly greater than 1 . This kind of notation will be used also in the sequel.

Remark 3.1. It is worth stressing that in all the procedure (cf. for instance estimates (3.5) and (3.9)) we are implicitly assuming that $\vartheta$ is positive at least almost everywhere. Actually, in [19] we were able to prove this property (and, actually, also a stronger one) by means of maximum principle arguments, even at the approximated level. In the current setting, due to the presence of singular terms on the right hand side of (2.8), ensuring the same property for approximate solutions is more delicate, requiring additional singular terms to be introduced in the process of approximation, see e.g. [21, Chapter 3].

\section{Estimate of the singular potential.}

The estimate of the (subdifferential of the) Ball-Majumdar potential constitutes the key point of our argument. Recalling estimate (3.13), we compute the $L^{2}$-scalar product of $\mathbb{H}$ with $-\vartheta \mathcal{L}\left[\frac{\partial f(\mathbb{Q})}{\partial \mathbb{Q}}\right]$. The necessary key properties of $f$ will be proved in the subsequent Section 4 . Hence, we have

$$
\begin{aligned}
I_{1}+I_{2} & :=\int_{\Omega} \vartheta^{2} \mathcal{L}\left[\frac{\partial f(\mathbb{Q})}{\partial \mathbb{Q}}\right]: \mathcal{L}\left[\frac{\partial f(\mathbb{Q})}{\partial \mathbb{Q}}\right]-\int_{\Omega} \vartheta \Delta \mathbb{Q}: \mathcal{L}\left[\frac{\partial f(\mathbb{Q})}{\partial \mathbb{Q}}\right] \\
& =-\int_{\Omega} \vartheta \mathbb{H}: \mathcal{L}\left[\frac{\partial f(\mathbb{Q})}{\partial \mathbb{Q}}\right]+\lambda \int_{\Omega} \vartheta \mathbb{Q}: \mathcal{L}\left[\frac{\partial f(\mathbb{Q})}{\partial \mathbb{Q}}\right]=: J_{1}+J_{2} .
\end{aligned}
$$

Moreover, by Hölder's and Young's inequalities and uniform boundedness of $\mathbb{Q}$ (cf. (3.8)),

$$
J_{2}=\lambda \int_{\Omega} \vartheta \mathbb{Q}: \mathcal{L}\left[\frac{\partial f(\mathbb{Q})}{\partial \mathbb{Q}}\right] \leq \frac{\kappa}{4}\left\|\vartheta \mathcal{L}\left[\frac{\partial f(\mathbb{Q})}{\partial \mathbb{Q}}\right]\right\|_{L^{2}\left(\Omega ; R_{\mathrm{sym}, 0}^{3 \times 3}\right)}^{2}+c(\kappa), \quad \kappa>0
$$

and

$$
J_{1}=-\int_{\Omega} \vartheta \mathbb{H}: \mathcal{L}\left[\frac{\partial f(\mathbb{Q})}{\partial \mathbb{Q}}\right] \leq \frac{\kappa}{4}\left\|\vartheta \mathcal{L}\left[\frac{\partial f(\mathbb{Q})}{\partial \mathbb{Q}}\right]\right\|_{L^{2}\left(\Omega ; R_{\mathrm{sym}, 0}^{3 \times 3}\right)}^{2}+c(\kappa)\|\mathbb{H}\|_{L^{2}\left(\Omega ; R_{\mathrm{sym}, 0}^{3 \times 3}\right.}^{2} .
$$

Notice that, integrated in time, the last term can be estimated by virtue of the first one in (3.13).

The key point is to control the term $I_{2}$ on the left hand side of (3.17). Using Green's formula, we have

$$
\begin{aligned}
I_{2} & =-\int_{\Omega} \vartheta \Delta \mathbb{Q}: \mathcal{L}\left[\frac{\partial f(\mathbb{Q})}{\partial \mathbb{Q}}\right] \\
& =\int_{\Omega} \vartheta \frac{\partial^{2} f(\mathbb{Q})}{\partial \mathbb{Q}_{i j} \partial \mathbb{Q}_{k l}} \nabla_{x} \mathbb{Q}_{i j} \cdot \nabla_{x} \mathbb{Q}_{k l}+\int_{\Omega}\left(\mathcal{L}\left[\frac{\partial f(\mathbb{Q})}{\partial \mathbb{Q}}\right]: \nabla_{x} \mathbb{Q}\right) \cdot \nabla_{x} \vartheta=: I_{2,1}+I_{2,2} .
\end{aligned}
$$

Then, thanks to the key estimate (4.7) below and (3.8),

$$
I_{2,1}=\int_{\Omega} \vartheta \frac{\partial^{2} f(\mathbb{Q})}{\partial \mathbb{Q}_{i j} \partial \mathbb{Q}_{k l}} \nabla_{x} \mathbb{Q}_{i j} \cdot \nabla_{x} \mathbb{Q}_{k l} \geq \varepsilon \int_{\Omega} \vartheta\left|\mathcal{L}\left[\frac{\partial f(\mathbb{Q})}{\partial \mathbb{Q}}\right]: \nabla_{x} \mathbb{Q}\right|^{2} .
$$


On the other hand, by Young's inequality,

$$
\left|I_{2,2}\right| \leq \frac{\varepsilon}{2} \int_{\Omega} \vartheta\left|\mathcal{L}\left[\frac{\partial f(\mathbb{Q})}{\partial \mathbb{Q}}\right]: \nabla_{x} \mathbb{Q}\right|^{2}+c(\varepsilon) \int_{\Omega}\left|\frac{\nabla_{x} \vartheta}{\vartheta^{1 / 2}}\right|^{2} .
$$

Then, integrating (3.17) over $(0, T)$, and taking $\kappa=\varepsilon / 2$, we get

$$
\left\|\vartheta \mathcal{L}\left[\frac{\partial f(\mathbb{Q})}{\partial \mathbb{Q}}\right]\right\|_{L^{2}\left((0, T) \times \Omega ; R_{\mathrm{sym}, 0}^{3 \times 3}\right)} \leq c,
$$

whence, comparing terms in $\mathbb{H}$ and using (3.8) and the first (3.13), we arrive at

$$
\|\Delta \mathbb{Q}\|_{L^{2}\left((0, T) \times \Omega ; R_{\mathrm{sym}, 0}^{3 \times 3}\right)} \leq c .
$$

Summarizing, we have obtained an $L^{2}$-control on both the Laplacian of $\mathbb{Q}$ and the singular term in (2.5).

\subsection{Weak sequential stability}

In this part, we assume to have a sequence $\left(\mathbf{u}_{n}, p_{n}, \mathbb{Q}_{n}, \vartheta_{n}\right)$ of weak solutions satisfying the estimates proved in the previous subsection uniformly with respect to $n$. Under these conditions, we show that there exists subsequence that converges in a suitable way to another weak solution to the problem. In order to simplify the notation, we assume all convergence relations appearing in the sequel to hold up to the extraction of (non-relabelled) subsequences.

First of all, notice that, thanks to the bounds (3.3)- 3.16 , we have

$$
\begin{aligned}
& \mathbf{u}_{n} \rightarrow \mathbf{u} \text { weakly star in } L^{\infty}\left(0, T ; L^{2}\left(\Omega ; \mathbb{R}^{3}\right)\right) \cap L^{\frac{2 A}{A+1}}\left(0, T ; W^{1, \frac{2 A}{A+1}}\left(\Omega ; \mathbb{R}^{3}\right)\right), \\
& \mathbb{Q}_{n} \rightarrow \mathbb{Q} \text { weakly in } L^{2}\left(0, T ; H^{2}\left(\Omega ; R_{\mathrm{sym}, 0}^{3 \times 3}\right)\right), \\
& \mathbb{Q}_{n} \rightarrow \mathbb{Q} \text { weakly star in } L^{\infty}\left(0, T ; H^{1}\left(\Omega ; R_{\mathrm{sym}, 0}^{3 \times 3}\right)\right) \cap L^{\infty}\left(0, T ; L^{\infty}\left(\Omega ; R_{\mathrm{sym}, 0}^{3 \times 3}\right)\right), \\
& \vartheta_{n} \rightarrow \vartheta \text { weakly star in } L^{\infty}\left(0, T ; L^{m}(\Omega)\right) \cap L^{A}((0, T) \times \Omega) \cap L^{2}\left(0, T ; H^{1}(\Omega)\right),
\end{aligned}
$$

the latter relation coming from (3.9) and interpolation. As a consequence of (3.13), we also obtain

$$
\mathbb{H}_{n} \rightarrow \overline{\mathbb{H}} \text { weakly in } L^{2}\left(0, T ; L^{2}\left(\Omega ; R_{\mathrm{sym}, 0}^{3 \times 3}\right)\right),
$$

for some tensor-valued function $\overline{\mathbb{H}}$. Here and below we use the upper bar to indicate weak limits that are not yet identified. In particular, we cannot express $\overline{\mathbb{H}}$ in terms of $\vartheta$ and $\mathbb{Q}$ at this level.

Next, by (3.26)-(3.27) and Gagliardo-Nirenberg inequalities (cf., e.g., [34]),

$$
\mathbb{Q}_{n} \rightarrow \mathbb{Q} \text { weakly in } L^{4}\left(0, T ; W^{1,4}\left(\Omega ; R_{\mathrm{sym}, 0}^{3 \times 3}\right)\right) .
$$

Then, by (2.6), (3.14),$A>9$ (cf. (2.13) $)$, and uniform boundedness of $\mathbb{Q}_{n}$,

$$
\left\|\mathbb{S}\left(\nabla_{x} \mathbf{u}_{n}, \mathbb{Q}_{n}\right)\right\|_{L^{\frac{9}{5}+}\left((0, T) \times \Omega ; R_{\mathrm{sym}, 0}^{3 \times 3}\right)} \leq c,
$$

whereas, by (3.16) and (3.30),

$$
\left\|\mathbf{u}_{n} \cdot \nabla_{x} \mathbb{Q}_{n}\right\|_{L^{\frac{12}{7}+}\left((0, T) \times \Omega ; R_{\mathrm{sym}, 0}^{3 \times 3}\right)} \leq c .
$$

By (3.59), (3.31), (3.32) and a comparison of terms in (2.5), we then infer

$$
\partial_{t} \mathbb{Q}_{n} \rightarrow \partial_{t} \mathbb{Q} \text { weakly in, say, } L^{\min \left\{\frac{12}{7}+, 2 A /(A+2)\right\}}\left((0, T) \times \Omega ; R_{\mathrm{sym}, 0}^{3 \times 3}\right) .
$$

Hence, by the Aubin-Lions lemma we get the following strong convergence relations:

$$
\begin{aligned}
& \mathbb{Q}_{n} \rightarrow \mathbb{Q} \text { in } L^{p}\left((0, T) \times \Omega ; R_{\mathrm{sym}, 0}^{3 \times 3}\right), \text { for all } p \in[1,+\infty), \\
& \nabla_{x} \mathbb{Q}_{n} \rightarrow \nabla_{x} \mathbb{Q} \text { in } L^{p}\left((0, T) \times \Omega ; R^{27}\right), \text { for all } p \in[1,4) .
\end{aligned}
$$


To proceed, we now compute the limit $n \nearrow \infty$ in the momentum equation. To start with, we first deal with the pressure $p$. This enters the total energy balance (2.17) as an additional unknown; hence, it needs to be estimated directly. To this purpose, one (formally) applies the operator div to $_{x}$ the momentum equation (2.3). This gives rise to an elliptic problem for $p$ which is well-posed thanks to the choice of periodic boundary conditions for all unknowns (cf. [12] for more details on this point). Recalling (2.4) and noting that

$$
\begin{aligned}
& \left\|\mathbf{u}_{n} \otimes \mathbf{u}_{n}\right\|_{L^{\frac{3}{2}+}\left((0, T) \times \Omega ; R^{3 \times 3}\right)}+\left\|\mu\left(\vartheta_{n}\right) \nabla_{x} \mathbf{u}_{n}\right\|_{L^{\frac{9}{5}+}{ }_{\left((0, T) \times \Omega ; R^{3 \times 3}\right)}} \leq c, \\
& \left\|\mathbb{H}_{n}\right\|_{L^{2}\left((0, T) \times \Omega ; R_{\mathrm{sym}, 0}^{3 \times 3}\right)}+\left\|\nabla_{x} \mathbb{Q}_{n} \odot \nabla_{x} \mathbb{Q}_{n}\right\|_{L^{2}\left((0, T) \times \Omega ; R^{3 \times 3}\right)} \leq c,
\end{aligned}
$$

thanks to (3.16), (3.25), (3.29) and (3.30), using also the uniform boundedness of $\mathbb{Q}_{n}$. It is then not difficult to deduce (cf. [18, Section 4] for more details)

$$
p_{n} \rightarrow p \text { weakly in } L^{\frac{3}{2}+}((0, T) \times \Omega) .
$$

Hence, a comparison of terms in (2.3) gives that

$$
\left\|\partial_{t} \mathbf{u}_{n}\right\|_{L^{\frac{3}{2}}+(0, T ; X)} \leq c
$$

where $X$ is a suitable Sobolev space of negative order. By the Aubin-Lions lemma and (3.16) we then get

$$
\mathbf{u}_{n} \rightarrow \mathbf{u} \text { strongly in } L^{3+}\left((0, T) \times \Omega ; R^{3}\right) .
$$

Hence, we have deduced strong (and consequently pointwise) convergence of $\mathbb{Q}_{n}$ and $\mathbf{u}_{n}$. The next step consists in obtaining an analogous property for the temperature. To this aim, we consider the total energy balance at the level $n$ and notice that

$$
\begin{aligned}
& \left\|\nabla_{x} \mathbb{Q}_{n}: \mathbb{H}_{n}\right\|_{L^{\frac{4}{3}\left((0, T) \times \Omega ; R^{3}\right)}} \leq c \text { by (3.29) and (3.30), } \\
& \left\|\sigma_{n} \mathbf{u}_{n}\right\|_{L^{1+}\left((0, T) \times \Omega ; R^{3}\right)} \leq c \text { by }(\text { (3.25), (3.38) }) \text { and (3.40), } \\
& \left\|\left|\mathbf{u}_{n}\right|^{2} \mathbf{u}_{n}\right\|_{\left.L^{1+}((0, T) \times \Omega) ; R^{3}\right)} \leq c \text { by (3.40). }
\end{aligned}
$$

Using (3.30), (2.6), 3.25) and uniform boundedness of $\mathbb{Q}_{n}$, we also get

$$
\left\|\nabla_{x} \mathbb{Q}_{n}: \mathbb{S}\left(\nabla_{x} \mathbf{u}_{n}, \mathbb{Q}_{n}\right)\right\|_{L^{\frac{36}{29}\left((0, T) \times \Omega ; R^{3}\right)}} \leq c .
$$

Moreover, using (3.28) and (2.13), we have

$$
\left\|\widehat{\kappa}\left(\vartheta_{n}\right)\right\|_{L^{1+}((0, T) \times \Omega)} \leq c, \text { provided that } A=\frac{3 k+2 m}{3}>k+1 \text {, i.e., } m>\frac{3}{2},
$$

the latter condition being a consequence of (2.13). Recall that $\widehat{\kappa}$ was defined in (2.18).

Now, it is clear that all summands in the internal energy $e_{n}$ (cf. (2.19) ) are uniformly $L^{2}$ bounded, with the exception of the last one for which we have

$$
\left\|\vartheta_{n}^{m}\right\|_{L^{\frac{3}{2}((0, T) \times \Omega)}} \leq c \text { provided that } \frac{3 k+2 m}{3} \geq \frac{3 m}{2}, \text { i.e., } 6 k \geq 5 m,
$$

which also follows from (2.13). By (3.46) and (3.16), we then find

$$
\left\|e_{n} \mathbf{u}_{n}\right\|_{L^{1+}\left((0, T) \times \Omega ; R^{3}\right)} \leq c .
$$

Collecting (3.41)-(3.47) and comparing terms in (2.17), we then infer

$$
\left\|\partial_{t}\left(\frac{1}{2}\left|\mathbf{u}_{n}\right|^{2}+e_{n}\right)\right\|_{L^{1+}(0, T ; X)} \leq c,
$$


where $X$ is, again, some Sobolev space of negative order. On the other hand, a direct computation based on (3.9), (3.11) and the other estimates permits to check that

$$
\left\|\nabla_{x}\left(\frac{1}{2}\left|\mathbf{u}_{n}\right|^{2}+e_{n}\right)\right\|_{L^{1+}\left((0, T) \times \Omega ; R^{3}\right)} \leq c, \text { provided that } \frac{3 k+2 m}{3}>2 m-k \text {, i.e., } 3 k>2 m,
$$

and also this condition is satisfied due to (2.13). Actually, we used here that

$$
\nabla_{x} \vartheta^{m}=c_{k, m} \vartheta^{m-\frac{k}{2}} \nabla_{x} \vartheta^{\frac{k}{2}} .
$$

With (3.48) and (3.49) at disposal, we can use once more the Aubin-Lions lemma to conclude that

$$
\left(\frac{1}{2}\left|\mathbf{u}_{n}\right|^{2}+e_{n}\right) \rightarrow\left(\frac{1}{2}|\mathbf{u}|^{2}+e\right) \text { strongly in } L^{1+}\left((0, T) \times \Omega ; R^{3}\right)
$$

and, consequently, almost everywhere in $(0, T) \times \Omega$. Note in particular that the limit of $\left|\mathbf{u}_{n}\right|^{2}$ is identified as $|\mathbf{u}|^{2}$ thanks to (3.40). Moreover, by (3.25), (3.28), and (3.27), we also have

$$
\left\|\frac{1}{2}\left|\mathbf{u}_{n}\right|^{2}+e_{n}\right\|_{L^{\infty}\left(0, T ; L^{1}(\Omega)\right)} \leq c .
$$

Hence, (3.48), (3.52) and a generalized form of the Aubin-Lions lemma give

$$
\left(\frac{1}{2}\left|\mathbf{u}_{n}\right|^{2}+e_{n}\right) \rightarrow\left(\frac{1}{2}|\mathbf{u}|^{2}+e\right) \text { strongly in } C^{0}([0, T] ; X),
$$

where $X$ is, again, some Sobolev space of negative order. Hence, the Cauchy condition for the total energy balance holds also in the limit, in the sense specified by (2.31). computing

By (3.51) and (3.40), we also obtain strong convergence of $e_{n}$, say, in $L^{1+}$. Consequently,

$$
\int_{0}^{T} \int_{\Omega}\left(e_{n}-e_{m}\right) \operatorname{sign}\left(\vartheta_{n}-\vartheta_{m}\right)
$$

for a couple of indexes $n, m$, and using monotonicity of the function $\vartheta \mapsto \vartheta^{m}$ (recall that the temperature is assumed to be nonnegative) and strong $L^{2}$-convergence of $\mathbb{Q}_{n}$ and $\nabla_{x} \mathbb{Q}_{n}$, we readily obtain that $\left\{\vartheta_{n}\right\}$ is a Cauchy sequence in $L^{1}((0, T) \times \Omega)$. Hence, recalling (3.28) and using a proper generalized version of Lebesgue's theorem, we arrive at

$$
\vartheta_{n} \rightarrow \vartheta \text { strongly in } L^{p}((0, T) \times \Omega) \text { for all } p \in[1, A) .
$$

As a consequence, thanks to assumptions (2.10)-(2.12), we infer

$$
\begin{aligned}
& \mu\left(\vartheta_{n}\right) \rightarrow \mu(\vartheta) \text { strongly in } L^{p}((0, T) \times \Omega) \text { for all } p \in[1, \infty), \\
& \kappa\left(\vartheta_{n}\right) \rightarrow \kappa(\vartheta) \text { strongly in } L^{p}((0, T) \times \Omega) \text { for all } p \in\left[1, \frac{3 k+2 m}{3 k}\right), \\
& \Gamma\left(\vartheta_{n}\right) \rightarrow \Gamma(\vartheta) \text { strongly in } L^{p}((0, T) \times \Omega) \text { for all } p \in[1, A) .
\end{aligned}
$$

Combining (3.28) and (3.29), and recalling assumption (2.12), we also get

$$
\Gamma\left(\vartheta_{n}\right) \mathbb{H}_{n} \rightarrow \overline{\Gamma(\vartheta) \overline{\mathbb{H}}} \text { weakly in } L^{\frac{2 A}{A+2}}\left(0, T ; L^{\frac{2 A}{A+2}}\left(\Omega ; R_{\mathrm{sym}, 0}^{3 \times 3}\right)\right) \text {. }
$$

Moreover, relation (3.58) also implies $\overline{\Gamma(\vartheta) \overline{\mathbb{H}}}=\Gamma(\vartheta) \overline{\mathbb{H}}$ (cf. also (3.59)). This permits us to pass to the limit in the momentum equation (2.3) (in particular, the stress $\sigma$ is identified in terms of the limit functions $\vartheta, \mathbb{Q}, \overline{\mathbb{H}}$ and $\mathbf{u}$ ). Analogously, we can take the limit in the Q-tensor equation (2.5) (where, however, the function $\overline{\mathbb{H}}$ is still to be identified) and in the total energy balance (2.17) (we use here also properties (3.44)-(3.48)).

In addition, from (3.55) and (3.10) we also obtain

$$
\log \vartheta_{n} \rightarrow \log \vartheta \text { strongly in } L^{p}((0, T) \times \Omega) \text { for all } p \in[1,2) .
$$


In particular, if positivity holds (almost everywhere) for $\vartheta_{n}$, then it is preserved in the limit $\vartheta$.

To conclude the proof, we need to take $n \nearrow+\infty$ in the relations involving the singular potential $f(\mathbb{Q})$. To be precise, what remains to do is identifying $\overline{\mathbb{H}}$ in terms of $\vartheta$ and $\mathbb{Q}$ and letting $n \nearrow \infty$ in the weak form (2.23) of the entropy production inequality.

We start with the first task. Thanks to estimate (3.23), we get that

$$
\vartheta_{n} \mathcal{L}\left[\frac{\partial f\left(\mathbb{Q}_{n}\right)}{\partial \mathbb{Q}_{n}}\right] \rightarrow \overline{\vartheta \mathcal{L}\left[\frac{\partial f(\mathbb{Q})}{\partial \mathbb{Q}}\right]} \text { weakly in } L^{2}\left((0, T) \times \Omega ; R_{\mathrm{sym}, 0}^{3 \times 3}\right) .
$$

Moreover, by strong convergence of $\vartheta_{n}$ and $\mathbb{Q}_{n}$ (cf. (3.55) and (3.34) $)$, and using that both $\mathbb{Q}_{n}$ and $\mathbb{Q}$ take their values almost everywhere into $\mathcal{D}[f]$ (otherwise $f\left(\mathbb{Q}_{n}\right)$ could not be uniformly $L^{1}$-bounded), we obtain

$$
\vartheta_{n} \mathcal{L}\left[\frac{\partial f\left(\mathbb{Q}_{n}\right)}{\partial \mathbb{Q}_{n}}\right] \rightarrow \vartheta \mathcal{L}\left[\frac{\partial f(\mathbb{Q})}{\partial \mathbb{Q}}\right] \text { a.e. in }(0, T) \times \Omega .
$$

By a generalized form of Lebesgue's Theorem, the combination of these facts entails that

$$
\vartheta_{n} \mathcal{L}\left[\frac{\partial f\left(\mathbb{Q}_{n}\right)}{\partial \mathbb{Q}_{n}}\right] \rightarrow \vartheta \mathcal{L}\left[\frac{\partial f(\mathbb{Q})}{\partial \mathbb{Q}}\right] \text { strongly in } L^{p}\left((0, T) \times \Omega ; R_{\mathrm{sym}, 0}^{3 \times 3}\right) \text { for all } p \in[1,2) .
$$

As a consequence of (3.63), $\overline{\mathbb{H}}$ is identified as $\mathbb{H}$, in terms of $\vartheta$ and $\mathbb{Q}$; namely, (2.7) holds. In particular, for $n \nearrow \infty$, equation (2.5) goes to the expected limit.

To conclude the proof, we have to take $n \nearrow \infty$ in the "weak" entropy production inequality (2.23). To this aim, we notice that, for any nonnegative-valued $\phi \in C_{c}^{\infty}([0, T) \times \Omega)$, there holds

$$
\begin{aligned}
\int_{0}^{T} & \int_{\Omega} \frac{\phi}{\vartheta}\left(\frac{\mu(\vartheta)}{2}\left|\nabla_{x} \mathbf{u}+\nabla_{x}^{t} \mathbf{u}\right|^{2}+\Gamma(\vartheta)|\mathbb{H}|^{2}+\frac{\kappa(\vartheta)}{\vartheta}\left|\nabla_{x} \vartheta\right|^{2}\right) \\
& \leq \liminf _{n \nearrow \infty} \int_{0}^{T} \int_{\Omega} \frac{\phi}{\vartheta_{n}}\left(\frac{\mu\left(\vartheta_{n}\right)}{2}\left|\nabla_{x} \mathbf{u}_{n}+\nabla_{x}^{t} \mathbf{u}_{n}\right|^{2}+\Gamma\left(\vartheta_{n}\right)\left|\mathbb{H}_{n}\right|^{2}+\frac{\kappa\left(\vartheta_{n}\right)}{\vartheta_{n}}\left|\nabla_{x} \vartheta_{n}\right|^{2}\right) .
\end{aligned}
$$

This is, indeed, a consequence of relations (3.25), (3.28), (3.29) (where, now, $\overline{\mathbb{H}}=\mathbb{H})$, of convexity of the above integrand with respect to $\nabla_{x} \mathbf{u}+\nabla_{x}^{t} \mathbf{u}, \mathbb{H}$, and $\nabla_{x} \vartheta$, and of a standard semicontinuity argument (see, e.g., [26]). Choosing $\phi \equiv 1$, we can manage the first integral in (2.23). Next, in order to deal with the second integral of (2.23), we simply observe that $-s$ is the sum of convex function both of $\vartheta$ and of $\mathbb{Q}$ and of the function $m a \vartheta^{m-1}$ (cf. (2.20)). Hence, we can take the (infimum) limit of that integral for a.e. $t \in(0, T)$ by virtue of relations (3.26)-(3.28) and (3.55), and of a further semicontinuity argument. This concludes the proof of Theorem 2.2

\section{Analytical properties of the Ball-Majumdar potential}

In this part we prove a number of fine properties of the Ball-Majumdar potential that were used in the proof. We recall first the definition and the basic features of the Ball-Majumdar potential. As Observed in [4, 5], the Ball-Majumdar singular potential $f$, defined as a function of $Q$, can be expressed as:

$$
f(Q)=F^{B M}\left(\lambda_{1}, \lambda_{2}, \lambda_{3}\right)
$$

where $\left(\lambda_{1}, \lambda_{2}, \lambda_{3}\right)$ are the eigenvalues of $Q$ and $F^{B M}$ is defined as

$$
F^{B M}\left(\lambda_{1}, \lambda_{2}, \lambda_{3}\right)=\sum_{i=1}^{3} \mu_{i}\left(\lambda_{i}+\frac{1}{3}\right)-\ln Z\left(\mu_{1}, \mu_{2}, \mu_{3}\right)
$$

with

$$
Z\left(\nu_{1}, \nu_{2}, \nu_{3}\right)=\int_{\mathbb{S}^{2}} \exp \left(\sum_{j=1}^{3} \nu_{j} p_{j}^{2}\right) \mathrm{d} p .
$$


and $\mu_{i}, i=1,2,3$, are given implicitly as solutions of the system:

$$
\frac{\partial \ln Z}{\partial \mu_{i}}=\lambda_{i}+\frac{1}{3}, i=1,2,3 \text {. }
$$

The solutions of (4.4) are determined up to an additive constant added to all the $\mu_{i}$ (i.e. if $\left(\mu_{1}, \mu_{2}, \mu_{3}\right)$ is a solution for a given triplet $\left(\lambda_{1}, \lambda_{2}, \lambda_{3}\right)$ then so is $\left(\mu_{1}+C, \mu_{2}+C, \mu_{3}+C\right)$ for any $C \in \mathbb{R})$.

Nevertheless, let us note that the $\mu_{i}, i=1,2,3$, can be taken, without loss of generality, as real analytic functions of $\left(\lambda_{1}, \lambda_{2}, \lambda_{3}\right)$. More precisely we have:

Lemma 4.1. There exist $\mu_{i}, i=1,2,3$, real analytic functions of $\left(\lambda_{1}, \lambda_{2}, \lambda_{3}\right)$, such that

$$
\sum_{i=1}^{3} \mu_{i}=0
$$

and solving the system (4.4).

Remark 4.2. The condition (4.5) is just a convenient way of eliminating the one-dimensional degeneracy in the solutions of the system (4.4).

Proof. We consider the linear space

$$
X=\left\{y \in \mathbb{R}^{3}: y \cdot(1,1,1)=0\right\} .
$$

We aim to apply the analytic implicit function theorem to $\mathcal{F}: X \times X \rightarrow X$ defined by

$$
\mathcal{F}\left(\lambda_{1}, \lambda_{2}, \lambda_{3}, \mu_{1}, \mu_{2}, \mu_{3}\right)=\left(\frac{\partial \ln Z}{\partial \mu_{1}}-\lambda_{1}-\frac{1}{3}, \frac{\partial \ln Z}{\partial \mu_{2}}-\lambda_{2}-\frac{1}{3}, \frac{\partial \ln Z}{\partial \mu_{3}}-\lambda_{3}-\frac{1}{3}\right)
$$

in order to express $\left(\mu_{i}\right)_{i=1,2,3}$ as functions of $\left(\lambda_{i}\right)_{i=1,2,3}$.

We note that $X$ is a 2 -dimensional space, with an orthonormal basis $f_{1}=\frac{1}{\sqrt{2}}(-1,1,0), f_{2}=$ $\frac{1}{\sqrt{6}}(1,1,-2)$. As such, in terms of this orthonormal basis, the non-degeneracy condition needed for applying the implicit function theorem is $\operatorname{det} M \neq 0$ for

$$
M=\left\{\begin{array}{ll}
\frac{\partial\left(\mathcal{F}, f_{1}\right)}{\partial f_{1}} & \frac{\partial\left(\mathcal{F}, f_{1}\right)}{\partial f_{2}} \\
\frac{\partial\left(\mathcal{F}, f_{2}\right)}{\partial f_{1}} & \frac{\partial\left(\mathcal{F}, f_{2}\right)}{\partial f_{2}}
\end{array}\right\}
$$

One can check that $\operatorname{det} M=36 \frac{\partial^{2} \ln Z}{\partial \mu_{1}^{2}} \frac{\partial^{2} \ln Z}{\partial \mu_{2}^{2}}-36\left(\frac{\partial^{2} \ln Z}{\partial \mu_{1} \mu_{2}}\right)^{2}$. It is convenient to consider the matrix

$$
N:=\left\{\begin{array}{cc}
\frac{\partial^{2} \ln Z}{\partial \mu_{1}^{2}} & \frac{\partial^{2} \ln Z}{\partial \mu_{1} \partial \mu_{2}} \\
\frac{\partial^{2} \ln Z}{\partial \mu_{1} \partial \mu_{2}} & \frac{\partial^{2} \ln Z}{\partial \mu_{1}^{2}}
\end{array}\right\}
$$

and observe that $\operatorname{det} M=36 \operatorname{det} N$. Then in order to check that $\operatorname{det} M \neq 0$ it suffices to check that for $e=\left(e_{1}, e_{2}\right) \in \mathbb{R}^{2}$ we have $(N e, e) \geq 0$ with equality if and only if $e=(0,0)$. Indeed, taking into account the definition (4.3) of $Z$ we have:

$$
(N e, e)=Z^{-2}\left[\left(\int_{\mathbb{S}^{2}} e^{\mu_{k} p_{k}^{2}}\left(p_{\alpha}^{2} e_{\alpha}\right)^{2} d p\right)\left(\int_{\mathbb{S}^{2}} e^{\mu_{k} p_{k}^{2}} d p\right)-\left(\int_{\mathbb{S}^{2}} e^{\mu_{k} p_{k}^{2}}\left(p_{\alpha}^{2} e_{\alpha}\right) d p\right)^{2}\right] \geq 0
$$

where for the last inequality we used Cauchy-Schwarz, and $k=1,2,3, \alpha=1,2$. It is clear that equality cannot hold for $e \neq(0,0)$ hence our claim.

We can now prove the fundamental estimate on the Hessian of $f$ : 
Proposition 4.3. There exists $\varepsilon>0$ such that:

$$
\frac{\partial^{2} f(\mathbb{Q})}{\partial \mathbb{Q}_{i j} \partial \mathbb{Q}_{k l}} \mathbb{V}_{i j} \cdot \mathbb{V}_{k l} \geq \varepsilon\left|\mathcal{L}\left[\frac{\partial f(\mathbb{Q})}{\partial \mathbb{Q}}\right]: \mathbb{V}\right|^{2} \quad \text { for all } \mathbb{Q} \in \mathcal{D}[f], \mathbb{V} \in R_{\mathrm{sym}, 0}^{3 \times 3}
$$

Proof. We proceed in several steps:

\section{Step 1: reduction to proving the concavity of an auxiliary function}

We set $h(\mathbb{Q}):=e^{-\varepsilon f(\mathbb{Q})}$ for all $\mathbb{Q} \in \mathcal{D}[f]$, where $\varepsilon>0$ is a positive constant to be chosen later on. It is more convenient to work with $h$ because the required property (4.7) is implied by $h$ being concave and the concavity of an isotropic function is in general simpler to check, as it will be seen in the next step.

We claim thus that $h$ being concave, smooth on $\mathcal{D}[f]$ and positive implies (4.7). We start by noting that for $\mathbb{Q} \in \mathcal{D}[f]$ and arbitrary $V \in R_{\text {sym }, 0}^{3 \times 3}$ :

$$
\begin{aligned}
\varepsilon \frac{\partial f(\mathbb{Q})}{\partial \mathbb{Q}_{i j}}: V_{i j} & =-\left.\frac{\mathrm{d}}{\mathrm{dt}}(\log h(\mathbb{Q}+t V))\right|_{t=0}=-\left.h(\mathbb{Q})^{-1}\left(\frac{\mathrm{d}}{\mathrm{dt}} h(\mathbb{Q}+t V)\right)\right|_{t=0} \\
& =-h(\mathbb{Q})^{-1} g(\mathbb{Q}, V)
\end{aligned}
$$

where we denoted $g(\mathbb{Q}, V):=\left.\frac{\mathrm{d}}{\mathrm{dt}} h(\mathbb{Q}+t V)\right|_{t=0}$.

Similarly, again for $\mathbb{Q} \in \mathcal{D}[f]$,

$$
\varepsilon \frac{\partial^{2} f}{\partial \mathbb{Q}_{i j} \partial \mathbb{Q}_{m n}} V_{i j} V_{m n}:=-\left.\frac{\mathrm{d}^{2}}{\mathrm{dt}^{2}} \log (h(\mathbb{Q}+t V))\right|_{t=0}=\frac{-k(\mathbb{Q}, V) h(\mathbb{Q})+g(\mathbb{Q}, V)^{2}}{(h(\mathbb{Q}))^{2}},
$$

where we denoted $k(\mathbb{Q}, V):=\left.\frac{\mathrm{d}^{2}}{\mathrm{dt}^{2}} h(\mathbb{Q}+t V)\right|_{t=0}$.

Then the desired estimate (4.7) is equivalent to

$$
\frac{g(\mathbb{Q}, V)^{2}-k(\mathbb{Q}, V) h(\mathbb{Q})}{(h(\mathbb{Q}))^{2}} \geq \frac{g(\mathbb{Q}, V)^{2}}{(h(\mathbb{Q}))^{2}}
$$

which clearly holds as $h>0$ and $k:=\left.\frac{\mathrm{d}^{2}}{\mathrm{dt}^{2}} h(\mathbb{Q}+t V)\right|_{t=0} \leq 0$ by our assumptions that $h$ is positive, smooth and concave.

The assumed positivity of $h$ is obvious (by the definition of $h$ ) and the smoothness is a consequence of the smoothness of $f$. We are left with checking the concavity.

\section{Step 2: checking concavity for a function of eigenvalues}

We define now $H:\left(-\frac{1}{3}, \frac{2}{3}\right) \rightarrow[0, \infty)$ by $H\left(\lambda_{1}, \lambda_{2}, \lambda_{3}\right):=h(\mathbb{Q})$ with $\mathbb{Q}$ having eigenvalues $\lambda_{i}, i=1,2,3$ (it is known that such a function is well defined and symmetric, see for instance [3] and the references therein). Then $h$ is concave if and only if $H$ is concave (see for instance Prop 18.2.4 in [44). We note that $h$ and $H$ have the same regularity (see for instance [3], Thm 5.5 for up to $C^{2}$ regularity and the extension up to $C^{\infty}$ in [45]). Thus $H$ is concave if and only if its Hessian is non-positive.

Step 3: the concavity of $H$ and the asymptotics of certain integrals

We have:

$$
\frac{\partial^{2} H}{\partial \lambda_{i} \partial \lambda_{j}}=\left(-\varepsilon \frac{\partial^{2} F^{B M}}{\partial \lambda_{i} \partial \lambda_{j}}+\varepsilon^{2} \frac{\partial F^{B M}}{\partial \lambda_{i}} \frac{\partial F^{B M}}{\partial \lambda_{j}}\right) e^{-\varepsilon F^{B M}} .
$$

Using the definition of $F^{B M}$ in (4.2), Lemma 4.1 and assuming $\mu_{i}, i=1,2,3$, to be differentiable functions of $\lambda_{i}, i=1,2,3$, we have: 


$$
\frac{\partial F^{B M}}{\partial \lambda_{j}}=\sum_{i=1}^{3} \frac{\partial \mu_{i}}{\partial \lambda_{j}}\left(\lambda_{i}+\frac{1}{3}\right)+\mu_{j}-\sum_{i=1}^{3} \frac{\partial \ln Z}{\partial \mu_{i}} \frac{\partial \mu_{i}}{\partial \lambda_{j}}=\mu_{j}, \forall j=1,2,3,
$$

where for the last equality we used (4.4).

On the other hand taking the partial derivative with respect to $\lambda_{j}, j=1,2,3$ in (4.4) we have:

$$
\sum_{k=1}^{3} \frac{\partial^{2} \ln Z}{\partial \mu_{i} \partial \mu_{k}} \frac{\partial \mu_{k}}{\partial \lambda_{j}}=\delta_{i j}
$$

Let us denote by $N=\left(N_{i j}\right)_{i, j=1,2,3}$ the matrix with components $N_{i j}:=\frac{\partial^{2} \ln Z}{\partial \mu_{i} \partial \mu_{j}}, i, j=1,2,3$. Then relations (4.94 4.10) show that

$$
\left(\frac{\partial \mu_{i}}{\partial \lambda_{j}}\right)_{i, j=1,2,3}=\left(\frac{\partial^{2} F^{B M}}{\partial \lambda_{i} \partial \lambda_{j}}\right)_{i, j=1,2,3}=N^{-1} .
$$

Hence, taking into account (4.8)-(4.10), in order to show that $H$ is concave we need to prove that for a suitable $\varepsilon>0$ the matrix:

$$
N^{-1}-\varepsilon \mu \otimes \mu
$$

is positive definite (where we denoted by $\mu \otimes \mu$ the matrix of components $\mu_{i} \mu_{j}, j=1,2,3$ ).

Let us recall now (see for instance [7, Proposition 1.2.6) that if $P, Q$ are real-valued symmetric matrices with $Q$ and $P Q+Q P$ positive definite then $P$ is positive definite as well. We aim to apply this criterion to $Q=N$ and $P=N^{-1}-\varepsilon \mu \otimes \mu$. To this end we need to show that $N$ is positive definite and

$$
\frac{2}{\varepsilon} \operatorname{Id}-(N \mu \otimes \mu+\mu \otimes \mu N) \text { is positive definite }
$$

for suitable $\varepsilon>0$. The proof of these two claims is quite technical and is postponed to the Appendix.

\section{The case of a singular heat flux law}

In this last section, we consider the case when the heat flux law exhibits a singular behavior for $\vartheta \sim 0$. Namely, in place of (2.11), we ask that

$$
\kappa(\vartheta)=A_{0}+A_{k} \vartheta^{k}+A_{-2} \vartheta^{-2}, A_{0}, A_{k}, A_{-2}>0 .
$$

Indeed other types of singular behavior may be considered as well. We chose the above expression since, as noted in the Introduction, it is in agreement with the behavior observed in various types of phase-transition and phase-separation models both related to liquids and to solids.

Mathematically speaking, the above choice permits to consider a more general class of singular potentials (still including the Ball-Majumdar case). Moreover, it permits us to get a stronger version of the entropy inequality (namely, it holds as a distributional inequality, and not only in the integrated form (2.23)). As a drawback, we have a marginal regularity loss for $\mathbb{Q}$. To state our related result, we first have to introduce the auxiliary function (cf. (2.11))

$$
H(\vartheta):=A_{0} \log \vartheta+\frac{A_{k}}{k} \vartheta^{k}-\frac{A_{-2}}{2} \vartheta^{-2},
$$

needed in the statement of the entropy inequality. 
Theorem 5.1. Let the coefficients $\mu, \kappa, \Gamma$ satisfy (2.10), (2.12), and (5.1), with relation (2.13) on exponents. Moreover, let the initial data comply with (2.14)-(2.16). Finally, let $f$ be any potential fulfilling conditions (i)-(iii) of Subsection 2.2 Then, there exists a quadruplet $(\mathbf{u}, p, \mathbb{Q}, \vartheta)$ with the regularity (2.24), (2.25) and (2.27), together with

$$
\begin{aligned}
& \mathbb{Q} \in L^{\infty}\left(0, T ; H^{1}\left(\Omega ; R_{\mathrm{sym}, 0}^{3 \times 3}\right)\right) \cap L^{9 / 5}\left(0, T ; W^{2,9 / 5}\left(\Omega ; R_{\mathrm{sym}, 0}^{3 \times 3}\right)\right), \quad \mathbb{Q}_{t} \in L^{\frac{18}{11}}\left((0, T) \times \Omega ; R_{\mathrm{sym}, 0}^{3 \times 3}\right), \\
& \log \vartheta \in L^{\infty}\left(0, T ; L^{1}(\Omega)\right) \cap L^{2}\left(0, T ; H^{1}(\Omega)\right), \quad \vartheta^{-1} \in L^{2}\left(0, T ; H^{1}(\Omega)\right),
\end{aligned}
$$

satisfying the incompressibility condition (2.2), the momentum equation (2.3), the Q-tensor evolution system (2.5) and the total energy balance (2.17) in the sense of distributions and complying with the initial conditions as specified in (2.30)-(2.31). Moreover, the entropy inequality holds in the distributional sense: for any nonnegative function $\phi \in \mathcal{D}([0, T] \times \bar{\Omega})$,

$$
\begin{aligned}
\int_{0}^{T} & \int_{\Omega} s \phi_{t}+\int_{0}^{T} \int_{\Omega} s \mathbf{u} \cdot \nabla_{x} \phi+\int_{0}^{T} \int_{\Omega} H(\vartheta) \Delta \phi \\
& \leq-\int_{0}^{T} \int_{\Omega} \frac{\phi}{\vartheta}\left(\frac{\mu(\vartheta)}{2}\left|\nabla_{x} \mathbf{u}+\nabla_{x}^{t} \mathbf{u}\right|^{2}+\Gamma(\vartheta)|\mathbb{H}|^{2}+\frac{\kappa(\vartheta)}{\vartheta}\left|\nabla_{x} \vartheta\right|^{2}\right) .
\end{aligned}
$$

Remark 5.2. Let us notice that under the current hypotheses, if we have any sufficiently smooth weak solution satisfying (5.5) with the equal sign, then that solution also satisfies the heat equation (2.8), or, in other words, it solves the problem in the "physical" sense. Hence, the current formulation and the physical one are in some sense equivalent. Instead, the same does not hold, even for smooth solutions, when we only know that the integrated entropy equality (i.e., (2.22) with the equal sign) holds.

Remark 5.3. Regarding the admissible class of potentials, we may relax even more our assumptions. Indeed, what we need for $f$ is simply that to be a convex function, with open bounded domain $\mathcal{D}[f]$, smooth inside $\mathcal{D}[f]$. Namely, we do not need $\mathcal{D}[f]$ to be related to the eigenvalues of $\mathbb{Q}$ (cf. (ii) of Subsection 2.2.

Proof of Theorem 5.1. The argument follows the lines of the proof given for Theorem 2.2. Hence, we only sketch the differences occurring in the a-priori estimates and in the weak stability argument.

First of all, we can observe that all the a priori bounds directly deriving from the energy and entropy balances still hold. Additionally, thanks to (5.1), (3.9) is improved as

$$
\left\|\nabla_{x} \vartheta^{\kappa / 2}\right\|_{L^{2}\left(0, T ; L^{2}\left(\Omega ; R^{3}\right)\right)}+\left\|\nabla_{x} \log \vartheta\right\|_{L^{2}\left(0, T ; L^{2}\left(\Omega ; R^{3}\right)\right)}+\left\|\nabla_{x} \vartheta^{-1}\right\|_{L^{2}\left(0, T ; L^{2}\left(\Omega ; R^{3}\right)\right)} \leq c .
$$

Combining the last of (5.6) with (3.4) and using a generalized version of Poincaré's inequality (cf. 42, Lemma 3.2] or [27, Lemma 5.1]), we deduce more precisely

$$
\left\|\vartheta^{-1}\right\|_{L^{2}\left(0, T ; H^{1}\left(\Omega ; R^{3}\right)\right)} \leq c .
$$

The key point, as before, is constituted by the estimate of the singular potential.

Estimate of the singular potential for singular heat flux. Differently from before, we do not need to rely on the explicit properties of the Ball-Majumdar potential. Indeed, we may directly compute

$$
\begin{aligned}
& \left\|\vartheta^{-1 / 2} \mathbb{H}\right\|_{L^{2}\left(\Omega ; R_{\text {sym }, 0}^{3 \times 3}\right)}^{2}=\int_{\Omega} \vartheta^{-1}|\Delta \mathbb{Q}|^{2}+\lambda^{2} \int_{\Omega} \vartheta^{-1}|\mathbb{Q}|^{2}+\int_{\Omega} \vartheta\left|\mathcal{L}\left[\frac{\partial f(\mathbb{Q})}{\partial \mathbb{Q}}\right]\right|^{2} \\
& \quad+2 \lambda \int_{\Omega} \vartheta^{-1} \Delta \mathbb{Q}: \mathbb{Q}-2 \int_{\Omega} \mathcal{L}\left[\frac{\partial f(\mathbb{Q})}{\partial \mathbb{Q}}\right]: \Delta \mathbb{Q}-2 \lambda \int_{\Omega} \mathcal{L}\left[\frac{\partial f(\mathbb{Q})}{\partial \mathbb{Q}}\right]: \mathbb{Q}=: \sum_{j=1}^{6} T_{j} .
\end{aligned}
$$


Now, let us observe that

$$
\begin{aligned}
\left|T_{4}\right| & =2 \lambda\left|\int_{\Omega} \vartheta^{-1} \Delta \mathbb{Q}: \mathbb{Q}\right| \leq \frac{1}{2} \int_{\Omega} \vartheta^{-1}|\Delta \mathbb{Q}|^{2}+2 \lambda^{2} \int_{\Omega} \vartheta^{-1}|\mathbb{Q}|^{2}, \\
T_{5} & =-2 \int_{\Omega} \mathcal{L}\left[\frac{\partial f(\mathbb{Q})}{\partial \mathbb{Q}}\right]: \Delta \mathbb{Q}=2 \int_{\Omega} \frac{\partial^{2} f(\mathbb{Q})}{\partial \mathbb{Q}_{i j} \partial \mathbb{Q}_{k l}} \nabla_{x} \mathbb{Q}_{i j} \cdot \nabla_{x} \mathbb{Q}_{k l}, \\
\left|T_{6}\right| & =2 \lambda\left|\int_{\Omega} \mathcal{L}\left[\frac{\partial f(\mathbb{Q})}{\partial \mathbb{Q}}\right]: \mathbb{Q}\right| \leq \frac{1}{2} \int_{\Omega} \vartheta\left|\mathcal{L}\left[\frac{\partial f(\mathbb{Q})}{\partial \mathbb{Q}}\right]\right|^{2}+2 \lambda^{2} \int_{\Omega} \vartheta^{-1}|\mathbb{Q}|^{2} .
\end{aligned}
$$

Then, integrating (5.8) in time over $(0, T)$ and using the latter of (3.13), we arrive at

$$
\begin{gathered}
\int_{0}^{T} \int_{\Omega} \vartheta^{-1}|\Delta \mathbb{Q}|^{2}+\int_{0}^{T} \int_{\Omega} \frac{\partial^{2} f(\mathbb{Q})}{\partial \mathbb{Q}_{i j} \partial \mathbb{Q}_{k l}} \nabla_{x} \mathbb{Q}_{i j} \cdot \nabla_{x} \mathbb{Q}_{k, l} \\
\quad+\int_{\Omega} \vartheta\left|\mathcal{L}\left[\frac{\partial f(\mathbb{Q})}{\partial \mathbb{Q}}\right]\right|^{2} \leq c+c \int_{\Omega} \vartheta^{-1}|\mathbb{Q}|^{2} \leq c
\end{gathered}
$$

the last inequality following from estimates (3.8) and (5.7). Hence, in place of (3.23)-(3.24) we get now

$$
\left\|\vartheta^{1 / 2} \mathcal{L}\left[\frac{\partial f(\mathbb{Q})}{\partial \mathbb{Q}}\right]\right\|_{L^{2}\left((0, T) \times \Omega ; R_{\mathrm{sym}, 0}^{3 \times 3}\right)}+\left\|\vartheta^{-1 / 2} \Delta \mathbb{Q}\right\|_{L^{2}\left((0, T) \times \Omega ; R_{\mathrm{sym}, 0}^{3 \times 3}\right)} \leq c .
$$

Using (3.11) and interpolation, we then obtain

$$
\left\|\vartheta \mathcal{L}\left[\frac{\partial f(\mathbb{Q})}{\partial \mathbb{Q}}\right]\right\|_{L^{\frac{2 A}{A+1}\left((0, T) \times \Omega ; R_{\mathrm{sym}, 0}^{3 \times 3}\right)}}+\|\Delta \mathbb{Q}\|_{L^{\frac{2 A}{A+1}\left((0, T) \times \Omega ; R_{\mathrm{sym}, 0}^{3 \times 3}\right)}} \leq c,
$$

where $\frac{2 A}{A+1}>\frac{9}{5}$ thanks to (2.13).

Weak sequential stability for singular heat flux. Also this part of the procedure goes through as before, the only difference being represented by some loss of regularity on $\mathbb{Q}$. Indeed, in place of (3.26), we now have, due to (5.14),

$$
\mathbb{Q}_{n} \rightarrow \mathbb{Q} \text { weakly in } L^{\frac{9}{5}}+\left(0, T ; W^{2, \frac{9}{5}+}\left(\Omega ; R_{\mathrm{sym}, 0}^{3 \times 3}\right)\right) .
$$

Combining (5.13) with the second (3.27) and using the Gagliardo-Nirenberg inequalities, it is not difficult to obtain

$$
\mathbb{Q}_{n} \rightarrow \mathbb{Q} \text { weakly in } L^{\frac{18}{5}+}\left(0, T ; W^{1, \frac{18}{5}+}\left(\Omega ; R_{\mathrm{sym}, 0}^{3 \times 3}\right)\right) .
$$

This leads to some obvious modifications in the exponents in the subsequent relations. For instance, in (3.32)-(3.33) $L^{12 / 7}$ turns to $L^{18 / 11}$; in the latter of (3.37), $L^{2}$ has to be replaced, say, by $L^{9 / 5}$; in (3.41), $L^{4 / 3}$ becomes $L^{9 / 7}$. Finally, $L^{36 / 29}$ is substituted by $L^{6 / 5}$ in (3.44). A further check shows that the argument used to get the strong convergence (3.53) still works even though the summability properties of the term $\left|\nabla_{x} \mathbb{Q}\right|^{2} / 2$ in the expression of $e(2.19)$ are now a little bit lower.

The subsequent convergence relations regarding $\vartheta$ do not change. Concerning, instead, the identification of the singular potential term, it suffices to notice that, in place of (3.61), we now have only

$$
\vartheta_{n} \mathcal{L}\left[\frac{\partial f\left(\mathbb{Q}_{n}\right)}{\partial \mathbb{Q}_{n}}\right] \rightarrow \overline{\vartheta \mathcal{L}\left[\frac{\partial f(\mathbb{Q})}{\partial \mathbb{Q}}\right]} \text { weakly in } L^{\frac{9}{5}}\left((0, T) \times \Omega ; R_{\mathrm{sym}, 0}^{3 \times 3}\right),
$$

due to (5.14).

To conclude the proof, we have to see that we can take the (supremum) limit in the distributional entropy inequality (5.5) (of course, we are now assuming it holds in that form at the level $n$ and want to show that it holds in the same form also in the limit). To this aim, we first notice that the right hand side is treated as in (3.64), taking now a generic (nonnegative) test function $\phi$. To proceed, we have to deal with the left hand side. We notice first that, by (5.7),

$$
\vartheta_{n}^{-1} \rightarrow \overline{\vartheta^{-1}} \text { weakly in } L^{2}\left(0, T ; H^{1}(\Omega)\right),
$$


whence, thanks to pointwise convergence (see (3.55)) and Sobolev's embeddings,

$$
\vartheta_{n}^{-1} \rightarrow \vartheta^{-1} \text { strongly in } L^{p}\left(0, T ; L^{q}(\Omega)\right) \text { for all } p \in[1,2), q \in[1,6) .
$$

In particular, we identify $\overline{\vartheta^{-1}}=\vartheta^{-1}$.

Next, let us note that, by (5.13), (5.18) and interpolation,

$$
\mathcal{L}\left[\frac{\partial f\left(\mathbb{Q}_{n}\right)}{\partial \mathbb{Q}_{n}}\right] \rightarrow \mathcal{L}\left[\frac{\partial f(\mathbb{Q})}{\partial \mathbb{Q}}\right] \text { weakly in } L^{4 / 3}\left(0, T ; L^{12 / 7}\left(\Omega ; R_{\mathrm{sym}, 0}^{3 \times 3}\right)\right) .
$$

Let us now recall the standard identity

$$
\mathcal{L}\left[\frac{\partial f(\mathbb{Q})}{\partial \mathbb{Q}}\right]: \mathbb{Q}=f(\mathbb{Q})+f^{*}\left(\mathcal{L}\left[\frac{\partial f(\mathbb{Q})}{\partial \mathbb{Q}}\right]\right),
$$

where $f^{*}$ is the convex conjugate (see, e.g., [40, Thm. 23.5, p. 218]) of $f$. Then, by (ii) of Subsection 22.2, $f^{*}$ grows at most linearly at infinity. Combining (5.20), (5.21) and (3.27), we then obtain

$$
f\left(\mathbb{Q}_{n}\right) \rightarrow f(\mathbb{Q}) \text { weakly in } L^{4 / 3}\left(0, T ; L^{12 / 7}(\Omega)\right) .
$$

Now, interpolating exponents in (3.25) and accounting for the strong convergence (3.40), we get

$$
\mathbf{u}_{n} \rightarrow \mathbf{u} \text { strongly in } L^{4+}\left(0, T ; L^{\frac{8}{3}+}\left(\Omega ; R^{3}\right)\right) \text {. }
$$

Moreover, we have

$$
\left\|\vartheta_{n}^{m-1}\right\|_{L^{\frac{3}{2}((0, T) \times \Omega)}} \leq c .
$$

Then, recalling the expression (2.20) for $s$, combining (5.22) with (5.23), and (5.24) and (3.60) with (3.40), we finally infer

$$
\begin{aligned}
& s_{n} \rightarrow s \text { say, strongly in } L^{1+}((0, T) \times \Omega), \\
& s_{n} \mathbf{u}_{n} \rightarrow s \mathbf{u} \text { strongly in } L^{1+}\left((0, T) \times \Omega ; R^{3}\right) .
\end{aligned}
$$

To conclude the proof, we need to take the limit in the third integral in the first row of (2.22), where we recall that $H$ was defined in (5.2). To this aim, we need a proper compactness tool:

Lemma 5.4. Let $h: \mathbb{R} \rightarrow[0,+\infty)$ be a continuous function such that $h(z) \rightarrow \infty$ as $z \rightarrow \infty$. Suppose that $\left\{u_{n}\right\}$ is a sequence of real-valued measurable functions defined over $(0, T) \times \Omega$ such that

$$
\begin{aligned}
& u_{n}(t, x) \geq 0 \text { for a.e. } x \in(0, T) \times \Omega, \\
& \text { ess } \sup _{t \in(0, T)}\left\|h\left(u_{n}\right)(t, \cdot)\right\|_{L^{1}(\Omega)} \leq H, \quad\left\|u_{n}\right\|_{L^{2}\left(0, T ; L^{p}(\Omega)\right)} \leq C \text { for some } p>2, \\
& u_{n} \rightarrow u \text { a.e. } \operatorname{in}(0, T) \times \Omega,
\end{aligned}
$$

for some positive constants $H$ and $C$. Then

$$
u_{n} \rightarrow u \text { strongly in } L^{2}((0, T) \times \Omega) .
$$

Proof. Clearly, it is enough to show equi-integrability of the $L^{2}$-norm of $u_{n}$, specifically

$$
\iint_{u_{n} \geq M}\left|u_{n}\right|^{2} \mathrm{dxdt} \rightarrow 0 \quad \text { as } M \rightarrow \infty .
$$

To see this, we first observe that

$$
H \geq \int_{u_{n}(t, \cdot) \geq M} h\left(u_{n}\right)(t, \cdot) \mathrm{dx} \geq \inf _{z \geq M} h(z)\left|\left\{u_{n} \geq M\right\}\right| \quad \text { for a.e. } t \in(0, T) .
$$

Now, applying the Hölder inequality, we get

$$
\int_{u_{n}(t, \cdot) \geq M} u_{n}^{2}(t, \cdot) \mathrm{dx} \leq\left|\left\{u_{n}(t, \cdot) \geq M\right\}\right|^{1 / q}\left\|u_{n}(t, \cdot)\right\|_{L^{p}(\Omega)}^{2}, \quad q=\frac{p}{p-2},
$$

whence (5.31) follows. 
Relations (3.4) and (5.18) allow us to apply the lemma with the choice $u_{n}=1 / \vartheta_{n}$ and $h(u)=\log u$. Hence, relation (5.19) is improved up to

$$
\vartheta_{n}^{-1} \rightarrow \vartheta^{-1} \text { strongly in } L^{2}((0, T) \times \Omega) .
$$

Hence, using (3.55), we have that

$$
H\left(\vartheta_{n}\right) \rightarrow H(\vartheta) \text { strongly in } L^{1}\left((0, T) \times \Omega ; R^{3}\right) .
$$

and consequently all terms on the left hand side of (5.5) pass to the limit $n \nearrow \infty$, which concludes the proof of the theorem.

\section{A A technical estimate}

We conclude the paper by providing the proof of the key relation (4.11), along with the necessary technical preparations. Our approach is based on the co-called Laplace's method to evaluate the integrals containing exponentials, see [25]. Let us first note that the positive definiteness of $N=$ $\left(\frac{\partial^{2} \ln Z}{\partial \mu_{i} \partial \mu_{j}}\right)_{i, j=1,2,3}$ is proved in [4. For convenience we recall the argument, namely:

$$
\begin{aligned}
\frac{\partial^{2} \ln Z}{\partial \mu_{i} \partial \mu_{j}} a_{i} a_{j} & =\frac{1}{Z} \int_{\mathbb{S}^{2}} e^{\sum_{k} \mu_{k} p_{k}^{2}} a_{i} p_{i}^{2} a_{j} p_{j}^{2} \mathrm{~d} p-\frac{1}{Z^{2}}\left(\int_{\mathbb{S}^{2}} e^{\sum_{k} \mu_{k} p_{k}^{2}} a_{i} p_{i}^{2} \mathrm{~d} p\right)\left(\int_{\mathbb{S}^{2}} e^{\sum_{k} \mu_{k} p_{k}^{2}} a_{j} p_{j}^{2} \mathrm{~d} p\right) \\
& =\frac{\int_{\mathbb{S}^{2}} e^{\sum_{k} \mu_{k} p_{k}^{2}} a_{i} p_{i}^{2} a_{j} p_{j}^{2} \mathrm{~d} p \int_{\mathbb{S}^{2}} e^{\sum_{k} \mu_{k} q_{k}^{2}} \mathrm{~d} q}{\int_{\mathbb{S}^{2}} \int_{\mathbb{S}^{2}} e^{\sum_{k} \mu_{k}\left(p_{k}^{2}+q_{k}^{2}\right)} \mathrm{d} p \mathrm{~d} q}-\frac{\int_{\mathbb{S}^{2}} e^{\sum_{k} \mu_{k} p_{k}^{2}} a_{i} p_{i}^{2} \mathrm{~d} p \int_{\mathbb{S}^{2}} e^{\sum_{k} \mu_{k} q_{k}^{2}} a_{j} q_{j}^{2} \mathrm{~d} q}{\int_{\mathbb{S}^{2}} \int_{\mathbb{S}^{2}} e^{\sum_{k} \mu_{k}\left(p_{k}^{2}+q_{k}^{2}\right)} \mathrm{d} p \mathrm{~d} q} \\
& =\frac{1}{2 Z^{2}} \int_{\mathbb{S}^{2}} \int_{\mathbb{S}^{2}} e^{\sum_{k} \mu_{k}\left(p_{k}^{2}+q_{k}^{2}\right)}\left(a_{i} p_{i}^{2}-a_{j} q_{j}^{2}\right)^{2} \mathrm{~d} p \mathrm{~d} q>0, \forall\left(a_{1}, a_{2}, a_{3}\right) \neq(0,0,0) .
\end{aligned}
$$

In estimating the positivity of $\frac{2}{\varepsilon} I d-(N \mu \otimes \mu+\mu \otimes \mu N)$ we consider the components of the matrix $N \mu \otimes \mu+\mu \otimes \mu N$, all functions of $\left(\mu_{1}, \mu_{2}, \mu_{3}\right) \in X$ (cf. (4.6)):

$$
\begin{aligned}
& \mathcal{I}_{i j}=\frac{\int_{\mathbb{S}^{2}} e^{\sum_{m} \mu_{m} p_{m}^{2}}\left(\sum_{k} \mu_{k} p_{k}^{2}\right)\left(\mu_{i} p_{j}^{2}+\mu_{j} p_{i}^{2}\right) \mathrm{d} p}{\int_{\mathbb{S}^{2}} e^{\sum_{m} \mu_{m} p_{m}^{2}} \mathrm{~d} p} \\
& -\left(\frac{\int_{\mathbb{S}^{2}} e^{\sum_{m} \mu_{m} p_{m}^{2}}\left(\sum_{k} \mu_{k} p_{k}^{2}\right) \mathrm{d} p}{\int_{\mathbb{S}^{2}} e^{\sum_{m} \mu_{m} p_{m}^{2}} \mathrm{~d} p}\right)\left(\frac{\int_{\mathbb{S}^{2}} e^{\sum_{m} \mu_{m} p_{m}^{2}}\left(\mu_{i} p_{j}^{2}+\mu_{j} p_{i}^{2}\right) \mathrm{d} p}{\int_{\mathbb{S}^{2}} e^{\sum_{m} \mu_{m} p_{m}^{2}} \mathrm{~d} p}\right) \\
& =\frac{\int_{\mathbb{S}^{2}} \int_{\mathbb{S}^{2}} e^{\sum_{m} \mu_{m}\left(p_{m}^{2}+q_{m}^{2}\right)}\left(\sum_{k} \mu_{k} p_{k}^{2}-\sum_{l} \mu_{l} q_{l}^{2}\right)\left[\mu_{i}\left(p_{j}^{2}-q_{j}^{2}\right)+\mu_{j}\left(p_{i}^{2}-q_{i}^{2}\right)\right] \mathrm{d} p \mathrm{~d} q}{2 \int_{\mathbb{S}^{2}} \int_{\mathbb{S}^{2}} e^{\sum_{m} \mu_{m}\left(p_{m}^{2}+q_{m}^{2}\right)} \mathrm{d} p \mathrm{~d} q} .
\end{aligned}
$$

It will suffice to show that $\mathcal{I}_{i j}\left(\mu_{1}, \mu_{2}, \mu_{3}\right), i, j=1,2,3$ is bounded independently of $\left(\mu_{1} \mu_{2}, \mu_{3}\right) \in$ $X$. To this end we consider two cases: when $\mu_{i} \neq \mu_{j}$ for $i \neq j$ and when two out the three $\mu_{i}, i=1,2,3$ are equal.

\section{A.1 Case I: $\mu_{i} \neq \mu_{j}$ for $i \neq j$}

We let $\mu_{i}=\rho \gamma_{i}$ with $\rho \geq 0$ and $\left(\gamma_{1}, \gamma_{2}, \gamma_{3}\right) \in \mathbb{S}^{2} \cap X$. It will suffice to prove:

$$
\lim _{\rho \rightarrow \infty} \mathcal{I}_{i j}\left(\rho \gamma_{1}, \rho \gamma_{2}, \rho \gamma_{3}\right)<\infty
$$

uniformly in $\left(\gamma_{1}, \gamma_{2}, \gamma_{3}\right) \in \mathbb{S}^{2} \cap X$.

We first recall the Laplace's asymptotic expansion method for evaluating integrals (see for instance [25] and also [8]), namely, let us consider the integral:

$$
J(h)=\int_{\Omega} e^{-h f(x)} g(x) d x
$$


with $\Omega \subset \mathbb{R}^{d}$ with $\Omega$ an open bounded set and $f, g \in C^{\infty}(\bar{\Omega}, \mathbb{R})$.

If $f$ has a single strict global minimum point in $\bar{x} \in \Omega$ such that $f(\bar{x})=0$ then for any $n \in \mathbb{N}$ one has:

$$
J(h)=\sum_{k=0}^{n} c_{k} h^{-\frac{k+d}{2}}+o\left(h^{-\frac{n+d}{2}}\right)
$$

as $h \rightarrow \infty$ with $c_{k}$ coefficients that are explicitly computable in terms of $f$ and $g$. More precisely we will only need the first three, $c_{0}, c_{1}, c_{2}$ given by

$$
\begin{gathered}
c_{0}=\Gamma\left(\frac{d+1}{2}\right) \int_{\mathbb{S}^{d-1}} \frac{g_{0}(\sigma)}{d\left(f_{0}(\sigma)\right)^{\frac{d}{2}}} \mathrm{~d} \sigma, \\
c_{1}=\Gamma\left(\frac{d+2}{2}\right) \int_{\mathbb{S}^{d-1}} \frac{2 d g_{1}(\sigma) f_{0}(\sigma)-(d+1) g_{0}(\sigma) f_{1}(\sigma)}{2 d(d+1) f_{0}(\sigma)^{\frac{d+3}{2}}} \mathrm{~d} \sigma, \\
c_{2}=\Gamma\left(\frac{d+3}{2}\right) \int_{\mathbb{S}^{d-1}} \frac{1}{d+2} \frac{g_{2}}{f_{0}^{\frac{d+1}{2}}}-\frac{f_{1} g_{1}}{(d+1) f_{0}^{\frac{d+4}{2}}}+\frac{f_{1}^{2} g_{0}}{4 d f_{0}^{\frac{d+6}{2}}}+\frac{\left(5 f_{1}^{2}-4 f_{0} f_{2}\right) g_{0}}{8 d f_{0}^{\frac{d+6}{2}}} \mathrm{~d} \sigma,
\end{gathered}
$$

where we denoted, for $\sigma=\frac{x}{|x|}$ :

$$
\begin{gathered}
f_{0}(\sigma):=\frac{1}{2}\left(\frac{\partial^{2} f}{\partial x_{i} \partial x_{j}}(\bar{x})\right)\left(\frac{(x-\bar{x})_{i}}{|x-\bar{x}|} \frac{(x-\bar{x})_{j}}{|x-\bar{x}|}\right), \\
f_{1}(\sigma)=\frac{1}{6}\left(\frac{\partial^{3} f}{\partial x_{i} \partial x_{j} \partial x_{k}}(\bar{x})\right)\left(\frac{(x-\bar{x})_{i}}{|x-\bar{x}|} \frac{(x-\bar{x})_{j}}{|x-\bar{x}|} \frac{(x-\bar{x})_{k}}{|x-\bar{x}|}\right), \\
g_{0}(\sigma)=g_{0}(\bar{x}), g_{1}(\sigma)=\frac{\partial g}{\partial x_{i}}(\bar{x}) \frac{(x-\bar{x})_{i}}{|x-\bar{x}|}, g_{2}(\sigma)=\frac{1}{2} \frac{\partial^{2} g}{\partial x_{i} \partial x_{j}}(\bar{x}) \frac{(x-\bar{x})_{i}}{|x-\bar{x}|} \frac{(x-\bar{x})_{j}}{|x-\bar{x}|} .
\end{gathered}
$$

Let us observe that in 25, the formulae were determined by allowing $g$ to be potentially singular at the maximum point. However, if $g$ is smooth (as is our case) then one can see that the odd-index coefficients $c_{2 k+1}$ are zero (see also [8] or directly compute $c_{1}$ by the formula above).

In order to apply the previous argument let us observe that the function $\gamma_{m}\left(p_{m}^{2}+q_{m}^{2}\right)$ of variable $(p, q):=\left(\left(p_{1}, p_{2}, p_{3}\right),\left(q_{1}, q_{2}, q_{3}\right)\right) \in \mathbb{S}^{2} \times \mathbb{S}^{2}$ attains its maximum value at two points, that depend on the maximum element in the set $\left\{\gamma_{1}, \gamma_{2}, \gamma_{3}\right\}$. Let us assume without loss of generality that $\gamma_{2}, \gamma_{3}<\gamma_{1}$. Then the maximum of the function $\gamma_{m}\left(p_{m}^{2}+q_{m}^{2}\right)$ is attained at two points $(p, p)$ with $p \in\{(1,0,0),(-1,0,0)\}$ so in order to apply Laplace's method we need to split $\mathbb{S}^{2} \times \mathbb{S}^{2}$ into two subdomains. Let us denote $\mathbb{S}_{E}:=\left\{p \in \mathbb{S}^{2}, p \cdot(1,0,0)>0\right\}$. We then apply the previously mentioned Laplace's method on each of the sets $\mathcal{E}:=\mathbb{S}_{E} \times \mathbb{S}_{E}$ and $\mathcal{V}:=\mathbb{S}^{2} \times \mathbb{S}^{2} \backslash \mathcal{E}$ chosing $h=\rho$ and $f\left(p_{1}, p_{2}, p_{3}, q_{1}, q_{2}, q_{3}\right):=\gamma_{1}-\gamma_{i}\left(p_{i}^{2}+q_{i}^{2}\right)$ (note that we can multiply both denominator and numerator in $\mathcal{I}_{i j}$ by $\left.e^{-\rho \gamma_{1}}\right)$.

The function $g$ will be chosen to be either $g \equiv 1$ (for treating denominators) or

$$
g_{i j}\left(p_{1}, p_{2}, p_{3}, q_{1}, q_{2}, q_{3}\right):=\left(\gamma_{k} p_{k}^{2}-\gamma_{l} q_{l}^{2}\right)\left[\gamma_{i}\left(p_{j}^{2}-q_{j}^{2}\right)+\gamma_{j}\left(p_{i}^{2}-q_{i}^{2}\right)\right]
$$

(for dealing with the numerators). Then we take $d=4$ and for treating the denominator we note that each of the two integrals (over $\mathcal{E}$, respectively $\mathcal{V}$ ) admit an asymptotic expansion of the form: $\bar{c}_{0} \rho^{-2}+o\left(\rho^{-2}\right)$. Similarily for the numerator the integrals have an expansion of the form $\rho^{2}\left(\tilde{c}_{0} \rho^{-2}+\right.$ $\left.\tilde{c}_{1} \rho^{-\frac{5}{2}}+\tilde{c}_{2} \rho^{-3}+\tilde{c}_{3} \rho^{-\frac{7}{2}}+\tilde{c}_{4} \rho^{-4}+o\left(\rho^{-4}\right)\right)$. As previously mentioned the coefficients of odd index, namely $\tilde{c}_{1}, \tilde{c}_{3}$ are zero. Taking into account the specific form of $g_{i j}$ (A.7) and the expressions of the coefficients $\tilde{c}_{0}$, namely (A.4) respectively $\tilde{c}_{2}$, namely (A.6) we have that both $g_{i j}$ and its first and second derivatives are zerd 1 so $\tilde{c}_{0}=\tilde{c}_{2}=0$. Thus the numerator will have an asymptotic expansion of the form $\tilde{c}_{4} \rho^{-2}+o\left(\rho^{-2}\right)$ and overall $\mathcal{I}_{i j}$ will be bounded as $\rho \rightarrow \infty$.

\footnotetext{
${ }^{1}$ note that the derivatives are computed at $(\theta, \varphi, \tilde{\theta}, \tilde{\varphi})=\left(0, \frac{\pi}{2}, 0, \frac{\pi}{2}\right)$ and we take $\left(p_{1}, p_{2}, p_{3}\right)=$ $(\cos \varphi \sin \theta, \sin \varphi \sin \theta, \cos \theta)$ with $(\varphi, \theta) \in[0,2 \pi] \times(0, \pi)$ and $\left(q_{1}, q_{2}, q_{3}\right)=(\cos \tilde{\varphi} \sin \tilde{\theta}, \sin \tilde{\varphi} \sin \tilde{\theta}, \cos \tilde{\theta})$ with $(\tilde{\varphi}, \tilde{\theta}) \in$ $[0,2 \pi] \times(0, \pi)$
} 


\section{A.2 Case II: two out the three $\mu_{i}, i=1,2,3$ are equal}

We assume without loss of generality that $\mu_{1}=\mu_{2}$ and we denote the common value by $\mu$. Then $\mu_{3}=$ $-\mu_{1}-\mu_{2}=-2 \mu$. After a couple of manipulations, using that $\left(p_{1}, p_{2}, p_{3}\right)=(\cos \varphi \sin \theta, \sin \varphi \sin \theta, \cos \theta)$ with $(\varphi, \theta) \in[0,2 \pi] \times(0, \pi)$ we have (by denoting $\left.\bar{Z}:=2 \pi \int_{0}^{\pi} e^{3 \mu \sin ^{2} \theta} \sin \theta d \varphi\right)$ :

$$
\begin{aligned}
& \mathcal{I}_{11}=\frac{6 \mu^{2}}{\bar{Z}} \int_{0}^{2 \pi} \int_{0}^{\pi} e^{3 \mu \sin ^{2} \theta} \cos ^{2} \varphi \sin ^{5} \theta \mathrm{d} \varphi \mathrm{d} \theta \\
& -\frac{12 \pi \mu^{2}}{\bar{Z}^{2}} \int_{0}^{\pi} e^{3 \mu \sin ^{2} \theta} \sin ^{3} \theta \mathrm{d} \theta \int_{0}^{2 \pi} \int_{0}^{\pi} e^{3 \mu \sin ^{2} \theta} \cos ^{2} \varphi \sin ^{3} \theta \mathrm{d} \varphi \mathrm{d} \theta \\
& \mathcal{I}_{22}=\frac{6 \mu^{2}}{\bar{Z}} \int_{0}^{2 \pi} \int_{0}^{\pi} e^{3 \mu \sin ^{2} \theta} \sin ^{2} \varphi \sin ^{5} \theta \mathrm{d} \varphi \mathrm{d} \theta \\
& -\frac{12 \pi \mu^{2}}{\bar{Z}^{2}} \int_{0}^{\pi} e^{3 \mu \sin ^{2} \theta} \sin ^{3} \theta d \theta \int_{0}^{2 \pi} \int_{0}^{\pi} e^{3 \mu \sin ^{2} \theta} \sin ^{2} \varphi \sin ^{3} \theta \mathrm{d} \varphi \mathrm{d} \theta \\
& \mathcal{I}_{33}=4 \mathcal{I}_{12}=4 \mathcal{I}_{21}=12 \mu^{2}\left[\frac{2 \pi}{\bar{Z}} \int_{0}^{\pi} e^{3 \mu \sin ^{2} \theta} \sin ^{5} \theta \mathrm{d} \theta-\left(\frac{2 \pi \int_{0}^{\pi} e^{3 \mu \sin ^{2} \theta} \sin ^{3} \theta \mathrm{d} \theta}{\bar{Z}}\right)^{2}\right] \\
& \mathcal{I}_{13}=\mathcal{I}_{31}=\frac{3 \mu^{2}}{\bar{Z}} \int_{0}^{2 \pi} \int_{0}^{\pi} e^{3 \mu \sin ^{2} \theta} \sin ^{5} \theta\left(-1-2 \cos ^{2} \varphi\right) \mathrm{d} \theta \mathrm{d} \varphi \\
& -\frac{6 \pi \mu^{2}}{\bar{Z}^{2}} \int_{0}^{\pi} e^{3 \mu \sin ^{2} \theta} \sin ^{3} \theta \mathrm{d} \theta \int_{0}^{2 \pi} \int_{0}^{\pi} e^{3 \mu \sin ^{2} \theta}\left(-1-2 \cos ^{2} \varphi\right) \sin ^{3} \theta \mathrm{d} \theta \mathrm{d} \varphi, \\
& \mathcal{I}_{23}=\mathcal{I}_{32}=\frac{3 \mu^{2}}{\bar{Z}} \int_{0}^{2 \pi} \int_{0}^{\pi} e^{3 \mu \sin ^{2} \theta} \sin ^{5} \theta\left(-1-2 \sin ^{2} \varphi\right) \mathrm{d} \theta \mathrm{d} \varphi \\
& -\frac{6 \pi \mu^{2}}{\bar{Z}^{2}} \int_{0}^{\pi} e^{3 \mu \sin ^{2} \theta} \sin ^{3} \theta d \theta \int_{0}^{2 \pi} \int_{0}^{\pi} e^{3 \mu \sin ^{2} \theta}\left(-1-2 \sin ^{2} \varphi\right) \sin ^{3} \theta \mathrm{d} \theta \mathrm{d} \varphi .
\end{aligned}
$$

Thus it suffices to understand what happens with ratios of the type $\frac{\int_{0}^{\pi} e^{3 \mu \sin ^{2} \theta}\left(\mu \sin ^{2} \theta\right)^{k} \sin \theta d \theta}{\int_{0}^{\pi} e^{3 \mu \sin ^{2} \theta} \sin \theta d \theta}$ for $k=1,2$. The case when $\mu \rightarrow \infty$ can be dealt with using Laplace's method previously described (but in $1 D$ now, as opposed to $2 D$ domains before), as in this case the maximum of $3 \sin ^{2} \theta$ occurs in the interior of the interval $(0, \pi)$. We obtain as desired that the limit as $\mu \rightarrow \infty$ of all the $\mathcal{I}_{i j}$ is finite.

In the case $\mu \rightarrow-\infty$ we note that $-3 \sin ^{2} \theta$ attains it maximum at the endpoints of the interval $(0, \pi)$ so Laplace's method cannot be applied directly. In this case we let $-\mu=\alpha^{2}$ and consider the change of variables: $\alpha \sin \theta=y$ which leads to:

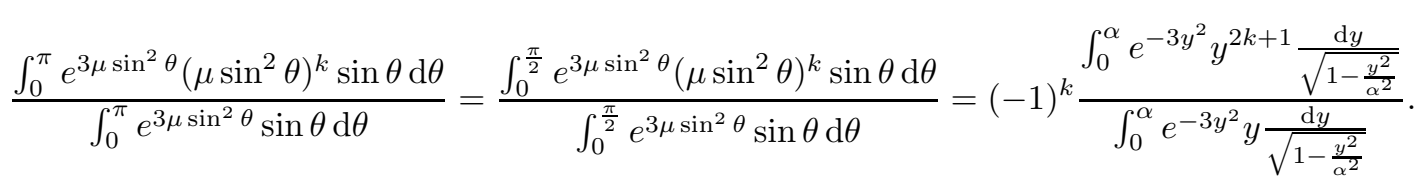

$$
\begin{aligned}
& \text { We let } f(\alpha):=\frac{\int_{0}^{\alpha} e^{-3 y^{2}} y^{2 k+1} \frac{\mathrm{d} y}{\sqrt{1-\frac{y^{2}}{\alpha^{2}}}}}{\int_{0}^{\alpha} e^{-3 y^{2} y} \frac{\mathrm{d} y}{\sqrt{1-\frac{y^{2}}{\alpha^{2}}}}} \text { and then we have: }
\end{aligned}
$$

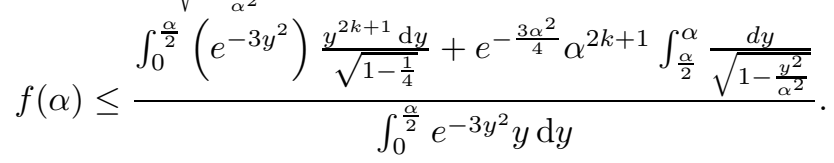


On the other hand:

$$
\begin{aligned}
& \int_{\frac{\alpha}{2}}^{\alpha} \frac{\mathrm{d} y}{\sqrt{1-\frac{y^{2}}{\alpha^{2}}}}=\int_{\frac{\alpha}{2}}^{\alpha} \frac{\alpha \mathrm{d} y}{\sqrt{(\alpha-y)(\alpha+y)}} \leq \frac{\alpha}{\sqrt{\frac{3}{2} \alpha}} \int_{\frac{\alpha}{2}}^{\alpha} \frac{\mathrm{d} y}{\sqrt{\alpha-y}} \\
& \leq \sqrt{\frac{2}{3}} \alpha^{\frac{1}{2}} \int_{0}^{\frac{\alpha}{2}} \frac{\mathrm{d} z}{\sqrt{z}}=\frac{2}{\sqrt{3}} \alpha .
\end{aligned}
$$

We assume without loss of generality that $\alpha>12^{2}$ and using this assumption as well as the last estimate in (A.8) we obtain:

$$
f(\alpha) \leq \frac{\int_{0}^{\infty}\left(e^{-3 y^{2}}\right) \frac{y^{2 k+1} \mathrm{~d} y}{\sqrt{1-\frac{1}{4}}}+e^{-\frac{3 \alpha^{2}}{4}} \alpha^{2 k+1} \frac{2}{\sqrt{3}} \alpha}{\int_{0}^{1 / 2} e^{-y^{2}} y \mathrm{~d} y},
$$

so $f(\alpha)$ is bounded independently of $\alpha$.

\section{References}

[1] H.W. Alt, I. Pawlow, A mathematical model of dynamics of non-isothermal phase separation, Physica D., 59 (1992), 389-416.

[2] J.-P. Aubin, Un théorème de compacité (French), C. R. Acad. Sci. Paris, 256 (1963), 5042-5044.

[3] J.M. Ball, Differentiability properties of symmetric and isotropic functions, Duke Math. J., 51, 1984, no. 3, 699-728.

[4] J.M. Ball, Mathematics of liquid crystals-short course, Cambridge Centre for Analysis, 2012,

[5] J.M. Ball, A. Majumdar, Nematic liquid crystals: from Maier-Saupe to a continuum theory, Molecular Crystals and Liquid Crystals, 525 (2010), 1-11.

[6] A.N. Beris, B.J. Edwards, Thermodynamics of Flowing Systems, Oxford University Press, Oxford, 1994.

[7] R. Bhatia, Rajendra, Positive definite matrices, Princeton Series in Applied Mathematics, Princeton University Press, Princeton, NJ, 2007.

[8] N. Bleistein, R.A. Handelsman, Asymptotic expansions of integrals. Second edition, Dover Publications, Inc., New York, 1986.

[9] D. Blanchard, O. Guibé, Existence of a solution for a nonlinear system in thermoelasticity, Adv. Diff. Eqns., 5 (2000), 1221-1252.

[10] E. Bonetti, P. Colli, M. Frémond, A phase field model with thermal memory governed by the entropy balance, Math. Models Methods Appl. Sci, 13 (2003), 1565-1588.

[11] E. Bonetti, M. Frémond, A phase transition model with the entropy balance, Math. Methods Appl. Sci., 26 (2003), 539-556.

[12] M. Bulíček, E. Feireisl, J. Málek, A Navier-Stokes-Fourier system for incompressible fluids with temperature dependent material coefficients, Nonlinear Anal. Real World Appl., 10 (2009), 9921015.

[13] C. Cavaterra, E. Rocca, On a 3D isothermal model for nematic liquid crystals accounting for stretching terms, Z. Angew. Math. Phys., 64 (2013), 69-82.

\footnotetext{
form

${ }^{2}$ that $f(\alpha)$ is bounded when $\alpha \in[0,1]$ can be more easily seen by looking at the expression of $f(\alpha)$ in trigonometric
} 
[14] P. Colli, Ph. Laurençot, Weak solutions to the Penrose-Fife phase field model for a class of admissible heat flux laws, Phys. D, 111 (1998), 311-334.

[15] P.G. De Gennes, J. Prost, The Physics of Liquid Crystals, Oxford University Press, Oxford, 1995.

[16] C. Denniston, J.M. Tóth, G. Yeomans, Lattice Boltzmann simulations of liquid crystal hydrodynamics, Phys. Rev. E, 63:056702 (2001).

[17] J. Ericksen, Liquid crystals with variable degree of orientation, Arch. Rational Mech. Anal., 113 (1991), 97-120.

[18] E. Feireisl, M. Frémond, E. Rocca, G. Schimperna, A new approach to non-isothermal models for nematic liquid crystals, Arch. Rational Mech. Anal., 205 (2012), 651-672.

[19] E. Feireisl, E. Rocca, G. Schimperna, A. Zarnescu, Evolution of non-isothermal Landau-de Gennes nematic liquid crystals flows with singular potential, Comm. Math. Sci., 12 (2014), 317-343.

[20] E. Feireisl, J. Málek, On the Navier-Stokes equations with temperature-dependent transport coefficients, Differ. Equ. Nonlinear Mech., Art. ID 90616 (2006), 14 pp. (electronic).

[21] E. Feireisl, A. Novotný, Singular Limits in Thermodynamics of Viscous Fluids, Birkhauser, Basel, 2009.

[22] E. Feireisl, E. Rocca, G. Schimperna, On a non-isothermal model for nematic liquid crystals, Nonlinearity, 24 (2011), 243-257.

[23] M. Frémond, Non-smooth Thermomechanics, Springer-Verlag, Berlin, 2002.

[24] A. Friedman, Partial Differential Equations of Parabolic Type, Prentice-Hall, Englewood Cliffs, NJ, 1964.

[25] W. Fulks and J.O. Sather, Asymptotics. II. Laplace's method for multiple integrals. Pacific J. Math., 11 (1961), 185-192.

[26] A.D. Ioffe, On lower semicontinuity of integral functionals. I, SIAM J. Control Optimization, 15 (1977), 521-538.

[27] N. Kenmochi, Neumann problems for a class of nonlinear degenerate parabolic equations, Differential Integral Equations, 3 (1990), 253-273.

[28] O.A. Ladyzhenskaya, V.A. Solonnikov, N.N. Uralceva, Linear and Quasilinear Equations of Parabolic Type, AMS, Trans. Math. Monograph 23, Providence, 1968.

[29] F.-H. Lin, C. Liu, Existence of solutions for the Ericksen-Leslie system, Arch. Rational Mech. Anal., 154 (2000), 135-156.

[30] J.L. Lions, Quelques Méthodes de Résolution des Problèmes aux Limites non Linéaires, Dunod, Gauthier-Villars, Paris, 1969.

[31] W. Maier, A. Saupe, A simple molecular statistical theory of the nematic crystalline-liquid phase, Z. Naturf., 14a (1959), 882-889.

[32] A. Miranville, G. Schimperna, Global solution to a phase transition model based on a microforce balance, J. Evol. Equ., 5 (2005), 253-276.

[33] N. Mottram, C. Newton, Introduction to Q-tensor theory, University of Strathclyde, Mathematics Research Report No. 10 (2004).

[34] L. Nirenberg, On elliptic partial differential equations, Ann. Scuola Norm. Sup. Pisa (3), 13 (1959), 115-162.

[35] M. Paicu, A. Zarnescu, Global existence and regularity for the full coupled Navier-Stokes and Q-tensor system, SIAM J. Math. Anal., 43 (2011), 2009-2049. 
[36] M. Paicu, A. Zarnescu, Energy dissipation and regularity for a coupled Navier-Stokes and Qtensor system, Arch. Rational Mech. Anal., 203 (2012), 45-67.

[37] O. Penrose, P.C. Fife, Thermodynamically consistent models of phase-field type for the kinetics of phase transitions, Phys. D, 43 (1990), 44-62.

[38] E. Rocca, R. Rossi, A degenerating PDE system for phase transitions and damage, preprint arXiv:1205.3578v2 (2013), 1-53, to appear on Math. Models Methods Appl. Sci., 2013.

[39] E. Rocca, G. Schimperna, Universal attractor for some singular phase transition systems, Phys. D, 192 (2004), 279-307.

[40] R.T. Rockafellar, Convex analysis, Princeton Mathematical Series, No. 28, Princeton University Press, Princeton, N.J. 1970.

[41] R. Rossi, T. Roubíček, Thermodynamics and analysis of rate-independent adhesive contact at small strains, Nonlinear Analysis 74 (2011), 3159-3190.

[42] G. Schimperna, A. Segatti, S. Zelik, Asymptotic uniform boundedness of energy solutions to the Penrose-Fife model, J. Evol. Equ., 12 (2012), 863-890.

[43] N. Schopohl, T.J. Sluckin, Defect core structure in nematic liquid crystals, Phys. Rev. Lett., 59, $2582-5$ (1987).

[44] M. Šilhavý, The mechanics and thermodynamics of continuous media, Texts and Monographs in Physics, Springer-Verlag, Berlin, 1997

[45] M. Šilhavý, Differentiability properties of isotropic functions, Duke Math. J., 104, 2000, no. 3, 367-373

[46] M. Wilkinson, Strict physicality of global weak solutions of a Navier-Stokes Q-tensor system with singular potential, arXiv:1211.6083v1 (2013), 1-26. 